Databases, Revenues, \& Repertory: The French Stage Online, 16801793 - Données, recettes \& répertoire: La scène en ligne (1680-1793)

\title{
Les sons du théâtre : \\ publics, acoustique, \\ déclamation
}

Juliette Cherbuliez

Published on: Oct 07, 2020

DOI: $10.21428 / 671 d 579 e . d c 3 a 8 c 7 c$

License: Creative Commons Attribution 4.0 International License (CC-BY 4.0). 
En découvrant le Projet des Registres de la Comédie-Française ( $\underline{\mathrm{RCF}}$ ), je me suis mise à parcourir ses pages numérisées et à imaginer un spectacle. Cet élan venait, je crois, de la curieuse manière dont, à chaque page, les rubriques imprimées se mêlent aux nombres inscrits à la main. Suivant le principe de tout formulaire, les catégories générales - Billets à... répété en haut de chaque page, systématiquement suivi par Frais journaliers, ou Pensions, Gages, \&c... - donnent une assise visuelle stable aux chiffres qui, eux, ne cessent de changer, indiquant le nombre de billets vendus, le prix des places et la date des représentations. Résistant à la tentation de me lancer dans une étude détaillée de ces données, sans doute par peur de faire des erreurs de calcul, j'ai instinctivement adopté une perspective à la croisée du formalisme et des performance studies pour tenter de comprendre cette danse des nombres qui virevoltent et se transforment à chaque page tournée. Ce spectacle assignait à chaque information un rôle au sein de l'ensemble plus large de la feuille. J'ai ainsi parcouru le registre R69 : 1715-1716 [Fig. 1].

\section{Visit the web version of this article to view interactive content.}

Figure 1. Registre R69, Saison 1715-16.

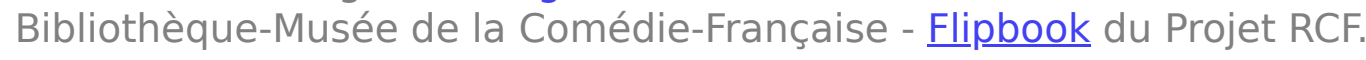

L'outil de visualisation m'apprend que je me trouve à la page 541 de ce volume et le registre indique qu'il s'agit de la $264^{\mathrm{e}}$ journée de la saison, « du jeudy $5^{\mathrm{e}}$ jour de mars 1716 première représentation d'Athalie ». J'imagine la foule des spectateurs jouant des coudes à l'entrée du Théâtre de la rue des Fossés Saint-Germain pour acheter leur billet : 4 loges basses à 40 livres ; 5 loges hautes à 20 livres ; 56 billets à 7 livres 10 sous ; 26 billets à 5 livres ; 66 billets à 3 livres ; 19 billets à 2 livres ; 290 billets à 1 livre 5 sous. Ces chiffres sont-ils l'indice d'une grande affluence ? On pourrait en tout cas présumer que le nombre de spectateurs est supérieur à celui de la veille, où la représentation de deux pièces de Molière n'a rapporté à la troupe que 97,10 livres. Je retrouve la même information dans le « double » de ce registre (R70), cette fois à la page 575 : la représentation de la même tragédie de Racine généra 1396 livres et 10 sous de recettes. Qu'est-ce que cette somme peut nous dire d'autre sur la représentation qui eut lieu ce soir-là ? Que nous apprend la répartition de ces billets qui correspondent à certaines catégories de places, assises ou debout, et à des zones plus ou moins bondées ? Qu'apportent ces éléments chiffrés - que l'on prenne en 
compte les nombres relatifs ou les quantités absolues - à l'étude de l'expérience globale de la séance théâtrale et de ses dynamiques ? À quoi ressemble un public qui rassemble 1396 livres et 10 sous, et que pouvons-nous savoir à son sujet ? Quelles sont les conséquences de sa présence sur la représentation, dans la mesure où les spectateurs ne se contentent pas de regarder la scène, mais qu'ils participent également au spectacle en influençant l'acoustique par leur présence corporelle?

Ceux qui ont un billet entrent dans le bâtiment. C'est la première représentation d'Athalie à la Comédie-Française. (S'agissait-il d'un événement attendu, ce qui expliquerait qu'Athalie ait été la seule pièce jouée cette soirée-là ? Les spectateurs auraient-ils toutefois été en droit d'attendre une représentation double avec, par exemple, une de ces comédies en un acte qui accompagnaient habituellement les tragédies ? Ou bien une tragédie chrétienne à machines de la fin du XVII ${ }^{\mathrm{e}}$ siècle étaitelle considérée comme l'équivalent d'une tragédie régulière accompagnée d'une comédie en un acte ?). Les spectateurs échangent leur billet pour une contremarque. (Avait-il connaissance de la pièce qui était représentée ? Était-ce important pour eux, ou se rendaient-ils au théâtre pour des raisons n'ayant rien à voir avec ce que la troupe présentait ?). Certains membres du public descendent vers le parterre et d'autres montent à l'étage pour rejoindre les loges. (Dans quelle mesure le billet à trois livres donnait-il accès à une meilleure place que celui qui en coûtait deux ? Combien y avait-il de loges et arrivait-il que certaines d'entre elles demeurent vides ?). On allume les bougies, on compte l'argent, et peut-être des coups de bâton annonçant le début du spectacle résonnent-ils.

Que se passe-t-il ensuite ? Dans l'expérience que me propose le Projet $\underline{\mathrm{RCF}}$, par l'intermédiaire de l'écran et du pavé tactile de mon ordinateur, un clic de souris permet d'avancer dans le temps. Les registres sont une archive des données recueillies quotidiennement, de sorte que chaque page respecte l'unité de temps aristotélicienne. À chaque clic, une nouvelle page, où la vision précédente disparaît : les spectateurs de cette représentation d'Athalie s'évanouissent. Vendredi, 6 mars 1716 : deux comédies sont au programme, Les Ménechmes et Le Médecin malgré lui ; aucune loge n’est réservée et à peine plus de deux cents billets sont vendus ce jour-là, pour une recette totale de 288 livres. Le public est moins nombreux que la veille. (Est-ce que les comédies vendent moins que les tragédies, ou est-ce Regnard et Molière qui n'attirent guère les spectateurs ? Les vendredis sont-ils plus calmes que les mercredis ? Y avaitil, le même jour, un autre événement qui a davantage séduit les spectateurs et les a tenus éloignés du théâtre ?). Un autre clic et la page se tourne de nouveau ; Athalie fait son retour et les recettes sont supérieures à celles qui avaient été engrangées 
deux jours plus tôt : 1995 livres, avec 404 billets à 1 livre 5 sous vendus. Est-ce là l'effet d'un bouche-à-oreille élogieux après la représentation précédente ? À chaque clic, le public va et vient, des billets se vendent, encore et encore. Il semble qu'on puisse identifier des récurrences dans les variations que connaissent l'affluence et les recettes quotidiennes. Les spectateurs sont attirés par des amis, des rivaux, une routine ; ils s'attendent à trouver des discours déclamés, de la musique, du pathétique, du spectacle sur scène ou dans la salle. C'est bien sûr la représentation de la pièce qu'ils viennent voir, mais leur attention est parfois détournée par une autre forme de spectacle ou de drame. C'est comme si je pouvais les voir aller et venir, par vagues successives.

Au fil des clics et des scènes imaginées se dessine comme un livre animé, ou une timelapse video, dans laquelle le temps et l'action avancent parfois au ralenti et parfois à toute vitesse, à la manière d'une plage où la marée semble monter et descendre plus ou moins rapidement selon son exposition aux vents. La scène est à peu de choses près la même chaque jour, même si costumes et spectateurs se transforment de plus en plus au fil des saisons et des années. Dans ce tableau, l'horizon est toujours le même, et toute interruption crée du rythme : chaque page suit le même modèle que la précédente et les exceptions sont à peine visibles. Les anomalies peuvent être néanmoins frappantes - comme par exemple quand je remarque ceci : des revenus particulièrement médiocres pour une soirée (un mercredi, le $18^{\mathrm{e}}$ jour de mars 1716 , L'École des femmes de Molière : 56,5 livres) ou une journée manquante (le mercredi 25 mars, dont l'absence est expliquée à la page suivante). Les pages sur lesquelles se trouve Athalie constituent une légère rupture. Lors des représentations de la tragédie, 500 livres « retiré[es] pour les frais de la pièce d'athalie » viennent s'ajouter aux dépenses habituelles de 30 livres pour les «lustres et decorations ». (Pourquoi les registres ne nous en disent-ils pas plus sur l'usage de ces 500 livres ? Il ne nous reste plus, alors, qu'à imaginer : étaient-elles dédiées à la machine révélant de manière si spectaculaire au dénouement le sanctuaire caché d'où Joas, le prince chrétien, sort pour être couronné, accompagné de soldats qui mettent un terme au règne d'Athalie? Le public a-t-il apprécié ces effets supplémentaires ?).

Est-ce du théâtre que j'imagine ? Dans ce cas, il faut en déterminer les éléments constitutifs les plus importants, et il ne s’agit pas des comédiens. La scène dans son ensemble se compose de la salle, avec ses sièges, ses couloirs, ses clivages, son hétérogénéité, ses habitudes, mais aussi les signes d'approbation ou d'ennui qui y apparaissent. Même quand l'action semble décousue, mon imagination reste concentrée sur le spectacle qu'offre le public, et non sur les comédiens eux-mêmes, 
dont le nom apparaît, parfois, au verso de la page et dans les registres des « feux » et des « assemblées ». Le public que j'imagine se transforme en une présence matérielle participant à ce que l'on pourrait appeler la para-performance, c'est-à-dire tous les éléments d'une représentation qui encadrent la scène : la foule désordonnée qui attend dehors pour acheter des billets, les spectateurs qui cherchent un siège, les interactions avec d'autres membres du public, ainsi que les réactions au spectacle, à la chaleur, au froid ou encore à la puanteur de la salle. Viennent ensuite les allées et venues : certains, peut-être un officier de police, sortent en plein milieu de la représentation, ou avant que la seconde pièce ne commence, ou finalement quand la séance s'achève. C'est donc principalement la dimension physique des spectateurs que j'imagine, même si les seules traces qui nous restent sont les sommes d'argent qu'ils ont déboursées pour entrer et que le Projet $\underline{\mathrm{RCF}}$ nous donnent à voir.

Malgré le rythme régulier des récurrences dans les registres, une fois que l'on a commencé à penser aux corps en mouvement et à leurs échanges, les scènes que nous imaginons sont bruyantes : s'ajoutent les sons ambiants et extra-diégétiques de spectateurs en train de manger, de danser, de se bousculer, de voler à la tire ou de se battre. Jeffrey Ravel a montré que tout cela faisait partie de l’expérience vécue par le spectateur de la première modernité - du moins dans le parterre. Bien différents de ceux des représentations de la seconde modernité à « la calme esthétique contemplative », ces éléments prouvent que « le chaos du parterre ne fait qu'augmenter l'intensité émotionnelle et intellectuelle de l'expérience des spectateurs 1 ». Quels étaient ces sons, dans leur relative diversité, jour après jour, représentation après représentation ? Comment pénétraient-ils la pièce qui se jouait, et que renvoyaient-ils aux acteurs et aux auteurs ? De quelle manière les bruits produits par le public influencent-ils le sens du théâtre, et comment le transformentils?

Tout cela nous rappelle à quel point la forme de la représentation théâtrale est le fruit de la rencontre entre des corps et des objets matériels : entre des acteurs et leur texte, entre des machinistes et leurs cordes, entre des spectateurs et le bâtiment même (mais aussi de façon tout aussi plausible : entre des acteurs et le bâtiment, entre le public et les cordes, et entre des machinistes et le texte). Mais où retrouver ces collisions dans une archive numérique ? Où se niche la texture sonore du théâtre ? Ainsi, un des dangers du Projet $\underline{\mathrm{RCF}}$ est qu'il semble réduire le théâtre au silence, le transformer en un espace muet et transactionnel qui pourrait se comprendre par le seul truchement de calculs. Toutefois, si, suivant les recherches des dix ou vingt 
dernières années, l'on conçoit la pratique théâtrale comme une entreprise s'adressant à tous les sens, et l'historiographie théâtrale, comme la tentative de retrouver les traces de ces perceptions dans les archives, comment dès lors intégrer à notre étude du Projet $\underline{\mathrm{RCF}}$ la multiplicité des sons qui étaient essentiels à la production culturelle de la Comédie-Française ? Que peuvent nous dire les nombres défilant sur les pages, au-delà du montant des bénéfices et des dettes ? Ne pourraient-ils pas nous donner un aperçu des individus dont chaque vente représenterait un corps et, plus précisément, nous en apprendre davantage sur la manière dont ces corps contribuent collectivement à l'événement théâtral ?

Dans quelle mesure visualiser les différents sens qui sont implicites dans les archives modifie-t-il non seulement notre conception de l'expérience théâtrale qu'elles recèlent, mais aussi nos méthodologies et nos pratiques historiographiques, ainsi que les objectifs qui les motivent ? En d'autres termes, dans quelle mesure une « resensorialisation » du passé du théâtre nécessite-t-elle une pratique différente des archives ? Peut-être nous faut-il repenser le sens politique de ces représentations, ainsi que nos méthodes, qui touchent aux questions de la compréhension, de la découverte, voire de la reconstitution du passé. Dès lors, quel sens donner aux bruits et aux sons qui sont recueillis par le Projet $\underline{\mathrm{RCF}}$ et que nous imaginons entendre, et comment cet exercice de l'esprit peut-il changer notre usage de cette base de données ?

Je voudrais proposer ici une expérience de pensée afin d'explorer les questions méthodologiques et historiographiques que soulève le travail sur les registres, compte tenu des recherches récentes sur l'histoire du théâtre. Celle-ci permettra d'ouvrir une piste insolite qui conduit à s'interroger non seulement sur le sens de la représentation théâtrale pendant la première modernité et sur sa dimension matérielle, mais aussi sur les avantages et les défis des enquêtes numériques en sciences humaines. Je souhaite penser le Projet $\underline{\mathrm{RCF}}$ en tant qu'archive sonore et explorer ce que permettrait la création d'un modèle acoustique modulable pour une pièce inscrite au répertoire de la Comédie-Française. J'espère ainsi mettre en lumière certaines possibilités parfois laissées de côté par les projets en humanités numériques.

\section{Pourquoi le son?}

Les liens entre théâtre et sons ont depuis longtemps été reconnus comme un des piliers de la représentation théâtrale - que ce soit par le biais des stratégies rhétoriques et déclamatoires, du rôle vital de la musique ou des questions d'acoustique 
et d'architecture $\underline{2}$. Dans la perspective propre à l'histoire du théâtre, l'attention prêtée aux sons ne se réduit pas à remettre en question la primauté du texte écrit dans les archives, mais elle modifie également l'histoire de l'esthétique et de la représentation associée à ces textes. La production de sons - depuis la voix des comédiens, le bruit des poulies des machinistes, les instruments de musique et les effets spéciaux faisant entendre le tonnerre, jusqu'aux interventions des spectateurs - est au cœur du théâtre.

De manière peut-être unique, le son contribue à un pan des histoires entrelacées de la représentation et du théâtre : il permet de répondre au récent appel à donner davantage de poids à ce que Christian Biet et Christophe Triau ont désigné comme « l’hétérogénéité, le champ de forces des instances » d'un théâtre où la présence dynamique et éphémère du public participe à ce(ux) qui crée(nt) la représentation $\underline{3}$. Admettre la prééminence du son dans la création d'un événement théâtral nécessite donc de considérer comme acteurs de la représentation des instances diverses, allant des comédiens qui déclament et des techniciens qui actionnent les câbles aux spectateurs qui se moquent, rient ou applaudissent, sans oublier ces objets qui amplifient le bruit et sans lesquels on n'entendrait rien. De la sorte, non seulement le son devient un élément important de la représentation et de l'interprétation que nous pouvons en faire, mais il apparaît aussi nécessaire de concevoir un modèle tout à fait différent de représentation. Le son est une dimension matérielle du théâtre à privilégier. On pourrait même défendre l'idée qu'il constitue une pierre angulaire pour développer un système théorique permettant de comprendre de manière globale la complexité sociale de la représentation théâtrale, créée par des interprètes et des techniciens, des membres du public et des acheteurs de billets, des machines faisant voler des personnages et des sièges qui grincent.

Si les modèles visuels dominants se sont surtout intéressés à des questions de politique de la représentation, en déterminant, par exemple, qui s'asseyait où et qui voyait quoi, les considérations sonores remettent fondamentalement en question la séparation entre les sens. Entendre, c'est aussi sentir : comme l'a montré l'archéologue des médias Wolfgang Ernst, le son est conçu comme un phénomène incarné depuis Archytas de Tarente, selon qui les phénomènes acoustiques sont d'ordre physique et non cognitif쓰. En outre, si l'on considère dans cette perspective la vaste palette des sons produits lors d'une représentation, il apparaît qu'ils sont le résultat d'un effort collectif : il n'y a qu'à considérer la manière dont les ondes sonores non seulement sont produites par des individus, mais aussi rebondissent sur les corps ou, au contraire, sont absorbés par eux, créant du lien entre celles et ceux qui 
occupent le même espace. On pourrait même considérer ces interactions comme des éléments cruciaux du «paysage sonore » du théâtre, terme inventé par le compositeur Murray Schafer, et désormais établi. Il désigne l'ensemble des sons qui définissent l'écologie acoustique d'un environnement à un certain moment et en un certain lieu, et auxquels s'ajoutent le travail et l'ordonnancement que nous opérons lorsque nous percevons, intégrons ou rejetons des sons en tant que « témoin auditif $\underline{5}$ ». Le paysage sonore du théâtre n'est pas le seul fait des comédiens, de la musique et des machines, ni même des spectateurs. Il est constitué du mélange des sons produits par ces instances et correspond donc au réseau que celles-ci créent avec l'espace qu'elles occupent. Le paysage sonore du théâtre est ce réseau de relations à proprement parler productives - réfléchissantes ou absorbantes -, établies entre des personnes, des objets matériels, le temps et l'espace, et pouvant être perçues et interprétées.

En pensant le théâtre à travers ses sons, on augment le nombre des agents - ou acteurs - qui contribuent à la création du spectacle. Les comédiens aussi bien que les spectateurs produisent des bruits qui influent sur l'expérience théâtrale. Les hautbois et les machines font de même, tout comme les colonnes qui réfractent, absorbent ou étouffent les ondes sonores. Une conception acoustique du théâtre implique de prendre en compte les individus et les groupes de gens agissant de concert ou, au contraire, en opposition, au sein d'un espace physique - avec ses matériaux et son espace propres - pour construire la représentation. Ainsi n'y a-t-il de sons au théâtre qu'en réseaux. Privilégier l'archive sonore du théâtre est donc une manière de développer encore davantage notre compréhension de ces réseaux constitutifs de l'expérience théâtrale et qui se trouvent, de manière sous-jacente, dans les documents que nous conservons.

J'essaie ici de répondre positivement à une question qu'a posée W. Ernst : «Y a-t-il un son de l'archive $\underline{6}$ ? » Toute enquête sur le son est amenée à suivre les réverbérations générées par les différents participants au sein des réseaux d'agents qui contribuent à sa production. Aussi le son est-il similaire à la performance dans la mesure où tous les deux se définissent en partie par leur dimension éphémère. Comme l'explique Peggy Phelan dans son analyse de référence, la performance « devient ce qu'elle est par sa disparition $\underline{7}$ ». N'existant que dans le moment où elle advient, la performance ne peut être déterminée en tant que telle qu'à partir du moment où elle se termine, disparait, a été et n'est plus. Si le son, à l'instar de l'événement théâtral, prend tout son sens par son évanescence, il constitue fondamentalement une catégorie historique. Ainsi conçu, le son, comme la performance telle que définie par P. Phelan, doit être envisagé alors 
qu'il s'évanouit. Notre analyse du phénomène sonore est toujours fondée sur des traces, qu'il s’agisse de la mémoire, des archives ou de preuves matérielles.

Paradoxalement, si le son est indispensable au théâtre, aucun son ne nous est parvenu de l'événement théâtral, toujours passé. Il y a cependant une histoire de ces sons. W. Ernst suggère que « les archives manuscrites et les textes imprimés laissent nécessairement de côté la question du son. Mais de manière plus profonde, il y a une sonorité implicite même dans les images, les diagrammes et les graphiques qui sont générés à partir de sources sonores. N'importe quel sonagramme conserve une relation indicielle avec l'événement sonore $\underline{8}$ ». Selon W. Ernst, toute représentation d'un son en constitue une archive et en contient la trace, quelle que soit la forme de cette archive.

W. Ernst se réfère ici aux cartographies réalisées par ultrasons, qui s’ajoutent aux partitions et autres enregistrements sous forme de diagramme. Peut-on étendre cette affirmation d'une « sonicité implicite » à un écrit rendant compte d'une dimension particulière de l'événement ? Autrement dit, est-il possible de concevoir le Projet $\underline{\mathrm{RCF}}$ comme l'archive d'un événement sonore ? Les registres représentent des performances et capturent, fût-ce de manière incomplète ou énigmatique, certains aspects d'un des éléments qui contribuent de manière décisive à l'environnement sonore lors d'une séance : le public. L'archive d'une représentation théâtrale enregistre presque littéralement - en-registre - d'une manière ou d'une autre les sons qui la constituent. C'est peut-être particulièrement vrai pour la première modernité : une pièce ne se matérialisait pas seulement au moment de la représentation mais circulait aussi sous forme imprimée, en partie pour pouvoir être déclamée dans des lieux de moindre taille. L'imprimé même est donc une sorte d'archive sonore, en particulier parce que le théâtre de cette époque s'appuie largement sur la tradition poétique complexe de l'art oratoire. Les conventions de publication des pièces de la période ont également partie liée avec l'oralité, comme il apparaît par exemple dans les pratiques des imprimeurs qui pouvaient se jouer des censeurs et du régime des privilèges en utilisant le script des acteurs ou la mémoire des spectateurs $\underline{9}$.

Ainsi, on pourrait soutenir que les registres sont avant tout une archive sonore, dans la mesure où ils proposent une représentation schématique d'un événement bruyant. Comme ils ne sauraient restituer le contexte sonore des spectacles, les données quotidiennes dessinent les contours de ce(ux) qui les produi(sen)t : les comédiens, les spectateurs, les portiers et les conducteurs des voitures, et les musiciens. Pour en revenir à ma première réaction qui inspira cet essai en me faisant imaginer le public 
d'une représentation, il semble désormais que c'est précisément l'absence de données sensorielles qui m’a conduite à considérer les données disponibles comme des archives d'événements. Que peut nous dire le Projet $\underline{\mathrm{RCF}}$, en tant qu'archive de performances évanescentes, du paysage sonore du théâtre ? Comment les données numériques des registres peuvent-elles permettre de concevoir le théâtre de la première modernité comme une expérience incarnée ? Une archive peut-elle jouer ? Quel sens donner à cette idée de faire performer une archive?

\section{L’archive imparfaite}

À ce stade, un lecteur raisonnable pourrait être rebuté par ces rêveries théoriques, voire spéculatives, au sujet d'une archive transformée en actrice d'une performance, et faire observer que ni ces archives en particulier ni cette période plus généralement ne se prêtent parfaitement à une enquête sur le son au théâtre.

Ce lecteur raisonnable pourrait également s'interroger sur l'utilité d'une telle projection en rappelant que des historiens du théâtre ont depuis longtemps pris en considération le son dans le théâtre du XVIII ${ }^{\mathrm{e}}$ siècle. Malgré l'absence d'enregistrements, certains chercheurs ont exploité les sources dont nous disposons et sont parvenus à une compréhension approfondie et nuancée des sons de la scène de la première modernité. En dépit des rares documents qui nous restent, ce que nous savons éclaire notre compréhension des arts du spectacle et de la culture française du $\mathrm{XVIII}^{\mathrm{e}}$ siècle ainsi que de la philosophie et des sciences des Lumières. Les recherches sur le son à la Comédie-Française ont mis en évidence les multiples dimensions d'une telle enquête, qui engage la diversité du public, l'acoustique des bâtiments et l’influence sur le théâtre de la philosophie matérialiste et de son sensualisme.

J. Ravel a identifié les personnes qui occupaient ces lieux, en montrant combien les membres du parterre étaient divers, tant par leur statut socioéconomique que par leur tempérament et leur respect des forces de l'ordre. Il souligne que l'expérience du parterre a évolué de façon dynamique tout au long du siècle, ce qui a de façon notable « permis au public de langue française du XVIII ${ }^{\mathrm{e}}$ siècle de développer un sens actif de la communauté, politique et générale, qui se différenciait des modèles défendus par la monarchie absolue ou par les élites sociales qui occupaient les bancs de scène et les loges $\underline{10}$ ». Comme le suggère le travail influent de J. Ravel, dès la première décennie du XVIII ${ }^{\mathrm{e}}$ siècle, l'intervention de membres du parterre durant la représentation a eu pour conséquence que les spectateurs sont devenus « définitivement des participants à 
la construction du sens de ce qui se jouait dans la salle de spectacle $\underline{11}$ ». L'installation de bancs en 1782 a été l'un des nombreux essais infructueux pour contrôler le parterre $\frac{12}{2}$ À défaut de parvenir à le dompter, cette entreprise eut un impact sur l'acoustique du théâtre même.

Définir les sons que pouvait produire le parterre et sa contribution sonore à l'acoustique d'ensemble du lieu constitue toutefois un autre problème. Tout d'abord, évaluer ce que les spectateurs de la Comédie-Française pouvaient entendre en général, et a fortiori durant une représentation précise, est particulièrement difficile parce que la troupe a occupé quatre bâtiments différents - sans compter que les sciences, la philosophie et l'importance de l'acoustique ont évolué au fil du $\mathrm{XVIII}^{\mathrm{e}}$ siècle. Ces changements nous donnent l'opportunité d'assister à la progressive transformation des idées concernant l'acoustique, comme l'ont montré les travaux de Jan Clarke et de Pannill Camp$\underline{13}$.

Dans son étude exhaustive sur l'histoire matérielle de la Comédie-Française pendant la première modernité, J. Clarke s'est penchée sur les deux premiers théâtres permanents : le théâtre Guénégaud et la Salle des Machines. Récemment, elle a étudié la plus ancienne Salle des Machines, qui fut utilisée occasionnellement au départ, avant de devenir un théâtre de pantomime, puis, plus tard, un lieu pour entreposer les décors. Elle a exploré des plans, des dessins et des rapports afin de comprendre pourquoi ce lieu avait la réputation d'avoir une acoustique exécrable et pourquoi le Jeu de paume a continué à être considéré comme un modèle possible. Différents facteurs, au nombre desquels figurent le souci de l'esthétique de la façade extérieure, la crainte liée aux matériaux inflammables et l'intérêt que pouvait avoir l'architecte à construire un espace impressionnant, ont contribué à la création d'un bâtiment dont les matériaux, l'énorme volume, les proportions et les embellissements décoratifs ont nui à l'acoustique. En concevant le théâtre comme le produit de diverses déterminations esthétiques, sociales et politiques, J. Clarke montre comment le succès - avec quelques échecs occasionnels - de la Comédie-Française a reposé de manière significative sur les matériaux utilisés pour la bâtir et sur l'espace où étaient jouées les pièces. La salle même devient une partie de la performance, un acteur à part entière. J. Clarke explique également de manière convaincante, en s'appuyant sur les théories de l'époque, pourquoi l'architecture tout en longueur du Jeu de paume connut un réel succès en tant que modèle acoustique. Selon J. Clarke, les problèmes de perspective et les limites imposées au spectacle que les spectateurs pouvaient donner d'eux-mêmes il est bien difficile de se donner à voir dans un Jeu de paume - furent acceptés parce 
que l'agencement de l'espace autorisait une excellente transmission du son. On peut alors se demander dans quelle mesure la prise en compte des problèmes acoustiques explique les nombreux changements dans la répartition des sièges dont témoignent les transformations des loges.

Ces questions d'acoustique ne se posaient pas seulement pour la structure des théâtres, mais elles étaient aussi soulevées dans les débats sur la nature de l'expérience. À la suite de Pierre Frantz $\frac{14}{14}$, qui suggère que la vogue du tableau théâtral est concomitante à la réévaluation des éléments visuels, comme les gestes, les poses, les costumes et les décors, Pannill Camp a montré de manière convaincante qu'il fallait revoir notre compréhension du rapport entre les évolutions de l'architecture théâtrale et les philosophies de l'expérience, qui eurent entre elles une influence réciproque, surtout durant les soixante dernières années du XVIII ${ }^{\mathrm{e}}$ siècle. Si l'on suit cette thèse, nous voyons que les questions touchant à l'optique et au visuel sont essentielles pour conceptualiser à la fois l'expérience théâtrale et ses influences sur la philosophie naturelle - ses espaces, ses méthodologies et ses spectateurs. P. Camp explique qu'au fil du siècle la philosophie sensualiste a contribué à faire émerger l'idée que les rapports entre le théâtre et le public touchent aux différents sens. Que ce soit au sujet de projets pour revoir l'architecture des théâtres, ou dans le cadre de débats sur l'illusion théâtrale ou sur la façon de jouer, les écrivains des Lumières en sont arrivés à mettre sur le même pied acoustique et visibilité. Dès 1765 , des architectes et des penseurs ont imaginé de nouveaux théâtres permettant « un acte spectatoriel dirigé vers la scène et mêlant audition et vision », et ont défendu le modèle d'une architecture théâtrale prenant en compte la voix du comédien, susceptible soit de « se perdre dans les coulisses » soit d'être trop forte, en particulier pour les spectateurs à proximité de la scène $\underline{15}$. Cependant, comme le remarque également $\mathrm{P}$. Camp, les traités et les croquis réalisés par les réformateurs du théâtre n'ont pas eu d'effet pratique immédiat sur les salles parisiennes. Quoi qu'il en soit, la physique expérimentale recommandait certaines précautions pour favoriser la diffusion des sons sur la scène tout en réduisant ceux produits par les spectateurs $\underline{16}$.

Ces travaux sont un appel à poursuivre de plus amples recherches sur le son au théâtre. Le Projet $\underline{\mathrm{RCF}}$ offre de manière évidente de remarquables ressources pour développer une analyse diachronique de ces espaces et de ces performances. Étant donné l'ampleur des données sur la vente des billets, il est tentant d'essayer de traquer dans ces données les changements sonores qu’a connus le théâtre sur un peu plus d'un siècle. Nous savons qu'il y a eu des transformations, ne serait-ce qu'à travers 
les évolutions des pratiques de jeu, des genres dramatiques et de l'acoustique théâtrale. Toutefois, l'analyse de ces transformations se heurte à des difficultés qui semblent insurmontables. En effet, la Comédie-Française a occupé quatre lieux différents au cours de cette centaine d'années - sans compter les représentations à la cour et dans d'autres salles - ce qui compromet, ou du moins complique, l'étude sur le long terme des évolutions du public. De plus, comme l'explique J. Clarke, les documents sur ces lieux sont rares et ceux qui nous restent sont si spécifiques à chaque salle que toute comparaison est difficile. En outre, J. Ravel a montré que les témoignages directs à propos de l'acoustique du théâtre sont de facto limités à leurs auteurs.

Peut-être faudrait-il nous intéresser à un cadre chronologique plus réduit pour gagner en cohérence. Si l'on considère les saisons, ou les représentations, les unes après les autres, le problème semble plus simple, même si le diable se loge toujours dans les détails, bien sûr. Représentation après représentation, les registres nous renseignent sur les billets vendus pour chaque catégorie de prix. S'il est vrai que l'on apprend par ce biais combien il fallait payer pour aller au théâtre, il est difficile d'établir le nombre exact de spectateurs présents dans la salle : les billets du parterre étaient individuels, mais pour les loges on déboursait un prix fixe et le nombre de personnes qu'elles contenaient pouvait donc varier. De plus, certaines d'entre elles étaient louées pour la saison, ce qui apparaît dans d'autres registres conservés dans les archives de la Comédie-Française. Enfin, leur capacité pouvait changer au fil des rénovations. Il faut donc distinguer les informations quantifiables de celles qui sont « moins exactes $\underline{17}$ ». Aucune inférence à partir d'une série de données n'a encore été vérifiée : on peut tenter de déterminer le taux d'occupation des loges à partir des ventes de billets pour le parterre, mais la validité de ce raisonnement n’a pas été démontré. Pour compliquer encore davantage les choses, il est difficile de déterminer comment la façon de tenir les registres a évolué au fil du siècle, compte tenu du fait que le nombre de catégories de billets n'a cessé d'augmenter : ces changements dans la répartition des places ontils ou non engendré un gain de précision dans la tenue des registres?

De quelle manière mesurer, comprendre et retracer les variations dans les pratiques comptables de la troupe ? Comment se servir de la vente des billets pour mieux comprendre l'impact des spectateurs qui les achetaient, entraient dans le théâtre et assistaient à la représentation ? Quelle était leur contribution à l'événement de la performance ? Était-il facile d'entendre quelque chose ? Quels étaient les effets acoustiques de la fréquentation, pour les spectateurs comme pour les comédiens ? Comment utiliser des données si diverses et fluctuantes pour déterminer avec une 
quelconque certitude les changements qui ont eu lieu ? Il suffit de considérer le type de preuves qui pourraient permettre d'initier cette enquête sur la sonicité des registres pour que les questions se multiplient. Avec chacune d'elle une nouvelle recherche s'ouvre ; et à chaque hypothèse, de nouvelles lacunes apparaissent dans les données, remettant en cause l'hypothèse même. Dans ces conditions, pourquoi vouloir modéliser la représentation comme un événement acoustique en s'appuyant sur une série de données qui est à la fois trop vaste et trop fragmentée, trop homogène et trop éparse?

\section{L'archive sonore comme un quasi-objet}

Peut-être est-ce précisément à partir de ces questions que font surgir ces archives aussi amples que désagréablement résistantes à l'interprétation, que l'on peut établir une enquête sur les sons du théâtre. Ces questions nous permettent en effet de concevoir différemment le champ d'étude ouvert par les registres. Étant donné la complexité des sons que suggèrent les traces présentes dans les registres, étant donné la multiplicité des acteurs (humains ou non) qui contribuent à la création d'un événement sonore, et étant donné la façon dont sujets et objets interagissent tout en dissimulant mutuellement leur présence dans les documents, il est possible que nous ayons là quelque chose qui excède un simple réseau de relations. Si l'on appréhende les registres comme une archive sonore, on est amené à en faire une sorte de sujet sujet qui génère des questions en série, qui réclame davantage de données et qui demande plus de modèles d'investigation. Ils ressemblent alors à ce que Bruno Latour désigne comme des «quasi-objets ». Le théoricien a eu tout d'abord recours à ce terme pour définir ce qu'il appelle des « hybrides », ces forces qui défient la division entre « nature » et « culture », entre « humain » et «non-humain » ou entre « sujet » et « objet ». Ces distinctions sont au fondement des modalités scientifiques d'interprétation de la connaissance et de la société, et elles assoient notre contrôle sur le monde qui nous entoure. B. Latour emprunte au philosophe Michel Serres le concept de quasi-objet pour décrire la façon dont les acteurs non-humains, à cheval sur la ligne de partage entre sujet et objet, ne se contentent pas de posséder une sorte d'agentivité, mais font également ressortir la qualité d’objet inhérente aux sujets qui entrent en contact avec eux $\underline{18}$.

La théorie de l'acteur-réseau (ANT) qu'a élaborée B. Latour, son rejet de celle-ci puis, plus tard, sa remobilisation et sa refonte dans Reassembling the Social $\underline{19}$ ont permis 
l'émergence d'une méthode d'enquête qui refuse l'idée fausse selon laquelle la Nature ou la société sont des objets en attente d'être découverts. Au lieu de cela, B. Latour défend l'idée que le social est une sorte d'assemblage temporaire et que nous devrions avoir pour but « d'enregistrer des connexions $\underline{20}$ ». Situés à l'intersection de la sociologie et de l'étude des sciences et des technologies (Science and Technology Studies), les travaux de B. Latour et de ses collègues sont à l'origine de pratiques pluridisciplinaires s'intéressant à la complexité de l'agentivité et de la production du sens, voire du monde. Ces pratiques reconfigurent des structures jusqu'alors considérées comme stables et permettent de refuser l'instauration de nouvelles entités de pouvoir.

La terminologie théâtrale à laquelle l'ANT recourt est également l'occasion de revenir sur une conception traditionnelle de la représentation comme matrice de la critique sociale, dans laquelle l'autorité critique n'est jamais remise en question. Utiliser le terme d'« acteur », par exemple, « implique que l'on ne simplifie pas trop vite celui qui passe à l'action, puisqu'un acteur sur scène n'est jamais seul à agir [...]. Le terme d'acteur dirige notre attention vers une redistribution complète de l'action et nous rappelle qu'elle n'est jamais une affaire cohérente, contrôlée, rondement menée, dont les contours seraient bien définis $\underline{21} »$.

Ici, l'usage de la terminologie théâtrale ne doit pas être confondu avec une métaphore filée ou une quelconque analogie. Il y a en effet une valeur épistémologique complexe dans cet appel à repenser d'où vient le savoir, et en particulier à examiner la manière dont les moyens par lesquels on retrace la production du savoir influent sur l'essence de cette dernière et sur ses potentialités. Dressant une « histoire de la performance océanique » qui intègre les théories indigènes de la conscience historique et l'altérité radicale des épistémologies polynésiennes, la chercheuse en théorie de la performance et en histoire du théâtre Margaret Werry soutient que l'ANT et le nouveau matérialisme constituent un cadre permettant de comprendre « la représentation ellemême non seulement comme un chemin d'accès à la connaissance, mais aussi comme une façon de connaître comment l'on arrive à connaître ${ }^{22}$ ».

Cette approche du réagencement épistémologique permis par l'ANT invite à penser que cette dernière est susceptible de modifier, de façon imprévue et radicalement imprédictible, nos méthodes et nos modes de savoir. Puisqu'il fait appel à des objets, à des matériaux de construction et à des personnes en contact les unes avec les autres dans un réseau d'échanges les poussant tous à œuvrer de concert, le théâtre ne saurait agir de façon indépendante. Au lieu de cela, il met en place son propre réseau 
de relations entre différents acteurs - personnes et piliers, poulies et bagarres, par exemple. La question du son et de la dynamique acoustique qui lui est corrélée fait apparaître cette dimension du théâtre comme quasi-objet. Si l'on considère le théâtre comme tel, admettant qu'il modifie les relations entre les corps vivants, les objets et l'espace, tout en étant transformé par ces derniers, on s'aperçoit que le son pourrait être un analogon approprié, ou une figure, pour la représentation théâtrale ainsi imaginée. Ce modèle suppose à bien des égards - cela va de soi - un lieu dynamique et actif : le théâtre est un quasi-objet quand il est en mouvement, en train de produire des sons et d'être, en retour, construit par eux. Dès lors, une archive peut-elle constituer un quasi-objet à son tour?

Cette question revient en partie à se demander si l'on peut légitimement conférer aux archives non seulement une agentivité, mais aussi la capacité de modifier leurs propres relations avec d'autres objets et d'autres sujets. Les vestiges du passé peuventils accomplir une telle tâche ? Loin de moi l'idée de me lancer dans quelques sinistres propos; je me refuse à utiliser des métaphores faciles comme celle de la possession, du retour à la vie ou de la résistance au temps $\underline{23}$, pour m'intéresser plutôt à ce qu'une archive sonore considérée comme quasi-objet pourrait faire spécifiquement. Ainsi conçue, l'archive apparaît comme une figuration monstrueuse, avec des pans nonexpliqués, étrangers et inutiles, avec des fonctions incompréhensibles, des informations qui peuvent aussi bien être erronées qu'indispensables à une compréhension future; elle ne représente pas tant le passé qu'elle offre une série de connexions entre des acteurs. Le quasi-objet qu'est l'archive met en action de supposés sujets et objets, et fait apparaître leurs associations, leurs influences réciproques et leurs effets. De la même manière que le son circule et qu'on étudie l'acoustique, l'archive comprise en tant que quasi-objet révèle des relations, voire crée des connexions. Loin de se réduire à un ensemble de documents prêts à être évalués et interprétés, elle compose plutôt une sorte de performance autoréflexive, dont la signification est peut-être susceptible de nous apparaître plus clairement si nous l'appréhendons non pas comme une base sur laquelle élaborer notre compréhension du passé, mais comme un interlocuteur qui participe à la définition des contours de notre enquête. Quand on lui laisse poser les questions, l'archive quasi-objet ne peut pas être seulement comprise comme un répertoire de preuves historiques ; d'une manière ou d'une autre, elle doit être impliquée dans l'enquête et dans les expériences dont elle est en même temps l'objet.

Une telle approche correspond peut-être à ce que B. Latour avait en tête quand il nous invitait à nous « nourrir des incertitudes » dans nos travaux et qu'il énumérait cinq 
sources possibles à celles-ci, insistant sur le refus d'une constitution ou d'une division arrêtées du pouvoir 24 . Si B. Latour s'attaque d'abord à une question actuelle - la prétendue objectivité de la sociologie et des disciplines connexes -, ses propositions peuvent également être appliquées aux approches historiques. Il suffit de considérer les réflexes de nombreux chercheurs travaillant en archives : leur quête d'une description historique à la précision idéale est paradoxalement accompagnée du lieu commun affirmant « qu’on ne peut jamais savoir ce qui s'est véritablement passé », ce qui autorise le projet initial de vouloir produire des « reconstructions ». Cette observation est particulièrement vraie en ce qui concerne la dernière source d'incertitude explorée par B. Latour : la recherche scientifique elle-même. Qu'elle ait la forme d'un article, d'une performance, d'un examen oral, d'un dossier ou d'un site internet, toute intervention scientifique doit se rendre disponible à la mise en question de ses pratiques instituées, et permettre que les principes qui la constituent soient réexaminés et traités de la même manière que le sont les objets d'étude auxquels elle s'intéresse. Elle doit être comprise non comme une conclusion, mais comme un « laboratoire », une expérience $\underline{25}$.

Quelle approche scientifique rendrait justice à une archive comprise comme un quasiobjet ? J'ai l'intuition que lui appliquer un modèle adapté pourrait nous permettre à la fois de mettre davantage en lumière les complexités du théâtre de la première modernité, et d'interroger de manière plus efficace ces archives, ainsi que les formes que nous leur donnons. Pour cela, il faudrait accepter leur caractère incomplet, tirer parti de leurs lacunes et de leurs manques, plutôt que de les effacer, et résister ainsi à l'envie de faire passer nos normes épistémologiques avant la compréhension de ce que théâtre, acoustique, performance, succès, sensation pouvaient alors signifier. Un tel modèle d'analyse irait jusqu'à chercher cette compréhension dans les failles et les ruptures des archives. Un modèle acoustique éviterait donc une définition positiviste de ce qu'il représente pour chercher plutôt à créer un cadre permettant de poser davantage de questions.

\section{Le laboratoire incertain}

Ce que je propose ici va à l'encontre de la plupart des modèles acoustiques historiques actuels, y compris et surtout les plus élaborés. Il est néanmoins utile de les prendre en considération et de les mettre en relation avec le Projet $\underline{\mathrm{RCF}}$ pour deux raisons. Premièrement, ils permettent de repérer les succès et les lacunes d'un modèle 
acoustique traditionnel. Deuxièmement, ils sont utiles pour mettre en évidence, par comparaison, la singularité du Projet $\underline{\mathrm{RCF}}$ et des possibilités qu'il offre en tant que terrain d'expérimentation.

Un des modèles les plus élaborés est sans doute celui du spécialiste de littérature anglaise John Wall, qui s'est intéressé au phénomène des prêches publics dans l'Angleterre de la Renaissance. Le modèle qu'il a développé nous apprend beaucoup de choses sur la conception d'un modèle acoustique modulable, aussi bien au niveau de l'archive même, que plus généralement au niveau historiographique. Il constitue une source d'inspiration quant à ce que la reconstitution du passé peut signifier et ce qu'elle peut offrir. Il constitue également une mise en garde.

À la différence de l'immensité des données du Projet $\underline{\mathrm{RCF}}$, J. Wall s'est intéressé à un objet singulier : le sermon que le prêcheur et poète John Donne a donné à la date anniversaire de la conspiration des Poudres (The Gunpowder Day Sermon). Pour soutenir le nouvel édit du roi Jacques $\mathrm{I}^{\mathrm{er}}$, s'opposant au prêche charismatique, Donne planifia un véritable spectacle, qui eut lieu le mardi 5 novembre 1622 à Saint-Paul's Cross, un espace extérieur de la cathédrale Saint-Paul dévolu aux prêches et pouvant accueillir jusqu'à 6000 personnes. J. Wall voulait saisir le sermon en tant que « phénomène social, occasion d'échanges et événement public $\underline{26}$ ». Au départ, son projet explorait une question bien précise qui ne concernait ni la culture de la Renaissance dans son ensemble ni le sermon de Donne, mais qui mettait en lien la performance de l'orateur et le rôle du public lors de la représentation : « combien de personnes pouvaient entendre une voix humaine non-amplifiée $\underline{27}$ ? » Avec cette question en tête, l'équipe dirigée par J. Wall commença par s'intéresser à l'acoustique et développa ensuite un modèle visuel.

Grâce à une bourse du National Endowment for the Humanities, les chercheurs développèrent le Virtual Paul's Cross Project (VPCP). Ils eurent recours à des modélisations architecturales, à des logiciels de simulation acoustique et à des comédiens pour proposer une expérience de cet événement, malgré les nombreuses variables que présentent les documents historiques. On ne connait l'église Saint-Paul, qui fut consumée par les flammes lors du grand incendie de Londres, qu'à travers des représentations picturales et quelques plans réalisés à partir de fouilles archéologiques. L'équipe demanda tout d'abord à un acteur de lire le sermon de Donne, long de deux heures, en respectant la prononciation du XVII ${ }^{\mathrm{e}}$ siècle. L'enregistrement eut lieu dans une chambre anéchoïque pour permettre des manipulations ultérieures. Les chercheurs prirent ensuite en compte les divers 
éléments pouvant affecter la représentation : la météo typique d'un jour de novembre londonien (couvert avec un vent léger), le smog causé par le charbon, les animaux présents (en particulier les chiens et les oiseaux) et les matériaux qui composaient le parvis de l'église (la pierre de la cathédrale et le bois des maisons avoisinantes) $\underline{28}$.

La Bibliothèque James B. Hunt de l'université de North Carolina Sate a accueilli ce projet, qui a été rendu public en 2013. Conçu comme un laboratoire technologique pour les résidents de la Caroline du Nord, le Teaching and Visualization Lab a permis de réaliser une installation constituée de vingt et une enceintes et dix projecteurs « afin de proposer une image continue à $270^{\circ}$ du parvis de Saint-Paul avec, pour ce qui concerne la dimension sonore, des sons d'ambiance favorisant l'immersion $\underline{29} »$. Le site du VPCP présente avec force détails la diversité des outils technologiques utilisés et la multitude des tâches réalisées. La modélisation visuelle a permis d'élaborer une armature pour le modèle acoustique afin de reconstruire le comportement des ondes sonores en fonction de l'endroit sur le parvis. De la sorte, si c'est une question d'acoustique qui était à l'origine du projet, la reconstitution visuelle du parvis disparu de Saint-Paul n'en a pas moins été une étape cruciale et fondatrice que l'on retrouve dans le rendu final.

La modélisation visuelle pour laquelle a opté le VPCP a été utilisée récemment par deux autres projets de reconstitution historique proposant une expérience virtuelle immersive : le PuddingLLane Project et le projet Rome Reborn, dont la première version a été achevée en 2007. Au début, les créateurs de ce second projet n’ont pas totalement embrassé l'idée d'une reconstitution historique, appelant leur travail un « modèle » ou « une représentation de l'état de nos connaissances (et, implicitement, de notre ignorance) $\underline{30} »$. La compagnie Flyover Productions est désormais propriétaire de Rome Reborn, maintenant décrit comme « une série de produits à destination des ordinateurs privés et des casques de réalité virtuelle et permettant de visiter la cité antique aujourd'hui disparue $\underline{31}$ ». Toute réflexion d'ordre épistémologique ou toute question concernant la spéculation historique ont disparu. À destination d'un public large, notamment constitué de touristes s'intéressant à Rome, le site vend une série d'applications et de vidéos proposant des visites guidées de la ville, ainsi que des questionnaires et un système d'évaluation des différents sites historiques sur les réseaux sociaux.

Qu'elles essaient de respecter l'histoire ou qu'elles s'en éloignent, ces reconstitutions reposent sur un lien avec des archives à partir desquelles il est possible d'élaborer une expérience se rapprochant de l'expérience d'origine. Elles sont également tributaires 
de la participation d'un public contemporain et de la façon dont il interagit avec le monde qu'elles créent. La grande popularité de la série de jeux Assassin's Creed, produit par Ubisoft, repose sur cette même technologie. Leur précision historique est telle que certains fans enregistrent et partagent leurs excursions en mélangeant extraits du jeu et vidéos tournées sur place. Peu importe que les joueurs d'Assassin's Creed incarnent virtuellement le corps d'un assassin qui voyage dans le temps, sautant et grimpant aux murs avec la force et les pouvoirs des héros Marvel. Certains de ces pèlerinages témoignent de recherches approfondies pour comparer les zones géographiques et les monuments réels à leur représentation dans le jeu ; ils en oublient souvent le caractère ludique et changeant du jeu au profit de l'action principale vécue par le héros, comme si l'on avait affaire à une reconstitution d'événements historiques. Ces jeux se proposent de représenter des mondes appartenant au passé de manière à ce que les joueurs puissent s'y projeter et les explorer comme s'ils constituaient des portails vers d'autres versions de l'histoire. Ces simulations numériques multi-sensorielles à grande échelle déjouent les stratégies de représentation du passé s'appuyant sur l'archéologie ou sur les faits historiques. Elles constituent ainsi des «polychronies »: des lieux où cohabitent des époques et des temporalités différentes et qui sont ainsi un «modèle de l'idée collective [du passé] que se fait le public contemporain $\underline{32} »$. Ni strictement historique ni complètement imaginaire, ces polychronies « existent dans un lieu étrange, à la frontière entre les deux, et mettent en relief les conséquences épistémologiques de certains modes de visualisation, tout en faisant communiquer entre eux des époques historiques, des événements et des lieux $\underline{33}$ ». Ces entreprises minutieuses ont permis aux historiens de reconsidérer les possibilités offertes par des mondes numériques dynamiques et immersifs. Selon Adam Chapman, les développeurs de jeux vidéo participent à une forme d'historiographie qui se rapproche du travail des historiens, qui privilégient pour leur part une « représentation écrite, empirique-analytique » et qui restent attachés à la «verticalité du livre ». Jouer aux jeux vidéo devient ainsi une pratique historiographique postmoderne à part entière $\underline{34}$.

Bien que les simulations des jeux vidéo se concentrent sur des mondes visuels, ce sont les mêmes principes de reconstitution du passé et les mêmes efforts pour créer une impression d'authenticité qui guident le Projet Bretez de Mylène Pardoen. Cette reconstitution du quartier du Grand Châtelet sous Louis XV s'intéresse surtout à l'environnement sonore mais a également recours à une modélisation visuelle en $3 \mathrm{D}$ 35. Cette dernière tend à être particulièrement séduisante pour les utilisateurs qui ont l'impression d'avoir à faire à une « reconstitution » fiable, mettant l'expérience en 
contexte. C'est peut-être pour cette raison que M. Pardoen - une musicologue - a inscrit les soixante-dix paysages sonores qu'elle a créés dans le cadre d'une promenade allant du pont au Change au pont Notre-Dame. Cette zone a été choisie pour son paysage sonore :

[I]l concentre 80\% des ambiances sonores du Paris de l'époque. Que ce soit à travers les activités qu'on y trouve - marchands, artisans, bateliers, lavandières des bords de Seine... -, ou par la diversité des acoustiques possibles, comme l'écho qui se fait entendre sous un pont ou un passage couvert... $\underline{36}$

La création de ces paysages sonore a certainement réclamé un travail considérable : M. Pardoen affirme qu'ils sont tous « naturels », y compris le son des machines, enregistré à partir d'appareils d'époque. Qualifier un son de naturel dans ce cas revient à souligner son authenticité et à indexer sa qualité sur celle du son original, que l'on a perdu et que l'on essaie d'imiter. M. Pardoen qualifie sa méthode d'« archéologie du passage sonore » et revendique son besoin de contrebalancer le travail d'archive par une activité de composition, plus esthétique et créative $\underline{37}$. La question de l'authenticité est passionnante, dans la mesure où, en tant que norme, elle exclut d'une certaine manière la singularité. En d'autres termes, M. Pardoen n'a pas reconstitué un son ou une journée en particulier, mais plutôt une ambiance sonore fidèle. Ainsi, le naturel ou l'authentique correspond ici au général.

Cette philosophie est à l'opposé de celle de Braxton Boren et Malcom Longair, par exemple, qui ont modélisé la musique renaissante d'une église vénitienne dans son lieu d'origine, ou encore de celle de Bissera V. Pentcheva, qui s'est intéressée à la basilique Sainte-Sophie, dans Icons of Sound. Ces paysages sonores privilégient le particulier pour parvenir à une authenticité provenant de la reconstitution d'un moment précis dans le temps. De manière analogue, Vincent Dumestre, Benjamin Lazar et Cécile Roussat ont reconstitué une représentation d'époque du Bourgeois gentilhomme (2004) grâce à un « vrai travail archéologique » : ils essayèrent de déterminer l'éclairage, la diction, le répertoire de gestes et les émotions que l'on trouvait dans une mise en scène du XVII ${ }^{\mathrm{e}}$ siècle ; certains critiques firent l'éloge de la façon dont cette représentation était éloignée de nos habitudes (et donc proche de l'expérience théâtrale du XVII ${ }^{\mathrm{e}}$ siècle), tandis que d'autres émirent des réserves sur la possibilité même d'une telle entreprise $\underline{38}$.

Paradoxalement, les modèles qui visent à reconstituer une ambiance générale recoupent ceux qui entendent faire revivre un moment particulier. Les uns comme les 
autres invitent leur public à comprendre ce que ce serait d'avoir assisté à un événement, d'y avoir participé, de l'avoir vécu, à un certain moment de l'histoire, dans un certain paysage sonore. D'une certaine manière, toutes ces entreprises investissent, voire cultivent la valeur attribuée à une certaine histoire spéculative. Mais là où l'approche traditionnelle de l'historiographie spéculative présente les étapes et les tendances permettant d'expliquer l'époque actuelle, voire ce vers quoi nous allons, le modèle expériential de la reconstitution historique suggère plutôt qu'il est possible de savoir ce qu'était ressentir tel ou tel moment $\underline{39}$. Ainsi, le balayage large que propose l'histoire spéculative et la modélisation sensorielle complexe qu'opèrent les créations historiques virtuelles se rejoignent, en cela qu'ils exercent une sorte de contrôle sur le passé afin de créer une relation continue et plaisante avec celui-ci. Un danger accompagne toute reconstitution historique : croire que l'on peut parfaitement retrouver le passé, que ce soit de façon générale ou particulière, implique souvent de proposer des détails qui ne se trouvent pas dans les archives.

Ces entreprises semblent se distinguer du VPCP, qui paraît singulièrement précis, voire unique, par sa portée limitée et sa méthodologie de recherche : le projet s'appuie sur un manuscrit existant pour représenter un sermon donné, prononcé à un moment précis et en un lieu déterminé. Peut-être cela tient-il à ce que Donne n'a finalement pas proféré son sermon dans le lieu en plein air qu'il avait en tête au moment de l'écriture et que l'équipe de J. Wall a pourtant reconstitué. En effet, l'événement fut déplacé dans la cathédrale « à cause du temps ». Si les textes de présentation du VPCP se gardent de mettre au premier plan ce fait historique, c'est en partie, selon moi, parce que cela va à l'encontre de l'idée que se font aussi bien les chercheurs que les institutions de financement de la recherche, qui est que le but du travail d’historien serait de révéler et de documenter ce qui s'est passé, et non ce qui n'a pas eu lieu.

De ce point de vue, on pourrait soutenir que J. Wall s'est livré à une sorte d'histoire spéculative singulière en recherchant un passé qui n'a jamais vraiment existé. C'est pourquoi on qualifie souvent ce type d'histoire exploratoire d'hypothétique, de virtuelle, de contrefactuelle. Que se serait-il passé si la France était devenue protestante, si la Révolution de 1830 s'était finie autrement, ou si la Révolution industrielle avait eu lieu principalement en France plutôt qu'en Angleterre $\underline{40}$ ? Alors que les historiens multiplient les débats sur la valeur des modèles qui explorent une version alternative du passé, je suis davantage intéressée par la manière dont ces exemples participent inévitablement à la valorisation d'une certaine téléologie, alors même qu'ils se proposent de la déconstruire. Nous sommes bien dans le régime de La Grande Histoire : celles des batailles, des grands bouleversements, de la naissance et 
de la disparition des cultures. Jamais les historiens contrefactuels ne proposeraient une histoire hypothétique d'un événement mineur : que ce serait-il passé si Rose Keller n'était pas rentrée avec le Marquis de Sade à la Pâque 1768 ? Et si Prévost n'avait pas publié le livre 7 de ses Mémoires et aventures d'un homme de qualité qui s'est retiré du monde séparément sous le titre de L'Histoire du chevalier Des Grieux et de Manon Lescaut? Que serait-il arrivé si Beaumarchais n'avait jamais fabriqué de montres ? Qu'ils soient spéculatifs ou virtuels, qu'ils résultent du travail d'un écrivain ou d'un ingénieur, les modèles historiques - sous forme de visualisations, de représentations acoustiques, ou encore, par exemple, de diagrammes - tendent à entretenir une vision de l'histoire comme progressant à la faveur d'événements d'envergure dont nous avons déjà établi l’importance.

Que serait-il arrivé si le Gunpowder Day Sermon avait effectivement été prononcé sur le parvis de Saint-Paul ? Bien que cette question ne soit pas totalement dénuée d'intérêt pour les historiens de la vie religieuse en Angleterre et les spécialistes de John Donne, on ne peut pas dire qu'elle apparaisse comme cruciale : elle ne saurait annoncer un changement fondamental dans notre connaissance de l'histoire. Depuis le départ, l'une des limites du VPCP est également l'une de ses forces : l'attention qu'il porte à un moment précis, qui n'a jamais eu lieu. Il examine de près les éléments constitutifs de ce sermon, de sa structure à son rythme en passant par sa réception acoustique et toutes les autres variables possibles. Il faut bien sûr remarquer que ce projet de grande ampleur, porté par plus de vingt-cinq personnes et financé par cinq organismes, dont le National Endowment for the Humanities, a abouti à la reconstitution d'un sermon. Cependant, la spécificité de l'entreprise constitue sa limite : une fois la reconstitution réalisée, le projet est achevé. Qu'apporte donc ce projet ? Est-ce que davantage d'enseignants incluront le Gunpowder Day Sermon dans leurs cours et est-ce qu'il permettra aux étudiants de mieux comprendre le phénomène du discours religieux ? Qui se soucie de savoir combien de personnes auraient pu assister au sermon sur le parvis?

Cependant, il est tentant de voir dans le VPCP un modèle virtuel contrefactuel ou hypothétique. J. Wall le qualifie de « restauration » et explique que ce projet n’a pas pour but de représenter un événement historique « mais un événement qui aurait dû se produire et qui finalement n'a jamais eu lieu avant aujourd'hui, 400 ans plus tard, dans un espace virtuel, certes, mais dans les deux heures qui lui avaient été imparties à l'origine. Ainsi, on comprend selon moi la valeur de la modélisation numérique, à la fois à un niveau spéculatif et en tant que restauration. Le projet permet de saisir ce 
qu'un tel modèle peut produire et le type de réalité qu'il établit $\underline{41}$ ». Loin de reconstituer le passé, la modélisation numérique répare une occasion manquée.

C'est peut-être pour cette raison que l'élément le plus intéressant du VPCP n'est pas son résultat final, c'est-à-dire sa performance virtuelle qui est à la fois éphémère (dans la mesure où elle ne se trouve plus là où elle a été créée à la North Carolina State University) et durable (grâce à l'archivage de ses éléments visuels et sonores). C'est plutôt le processus d'élaboration de cette expérience qui semble primordial, à tel point qu'une bonne partie du site internet est consacré à rendre compte de ce cheminement. Il consigne également les résultats inattendus qui sont apparus au fur et à mesure du développement du projet. Parce que J. Wall et son équipe n’ont pas essayé de reconstituer un moment particulier mais un événement qui n’a jamais eu lieu, ce projet permet « de rendre pleinement compte de l'ambiguïté et du caractère indéterminé d'une archive », tout en mettant en lumière ce que savent les historiens sur les prêches en plein air à Saint-Paul's Cross. La réalité que construit la modélisation numérique n'est pas seulement spéculative, elle est aussi générative.

L'équipe du VPCP a élaboré un modèle qui met au premier plan ces deux dimensions et propose une représentation du sermon accompagnée de sons d'ambiance - bruits d'eau, aboiements de chiens, coups de cloche - en fonction de certaines variables : il est possible de modifier la taille de la foule - de 250 à 5000 personnes - et de choisir différentes positions sur le parvis [Fig. 2]. Ainsi, la modélisation nous interdit de faire l'expérience de ce sermon sous la forme d'un événement singulier, pour nous pousser au contraire à prendre en compte la manière dont la position géographique (et donc sociale, selon que l'on appartient ou non à l'élite) modifie en profondeur la réception et la façon d'y participer. 


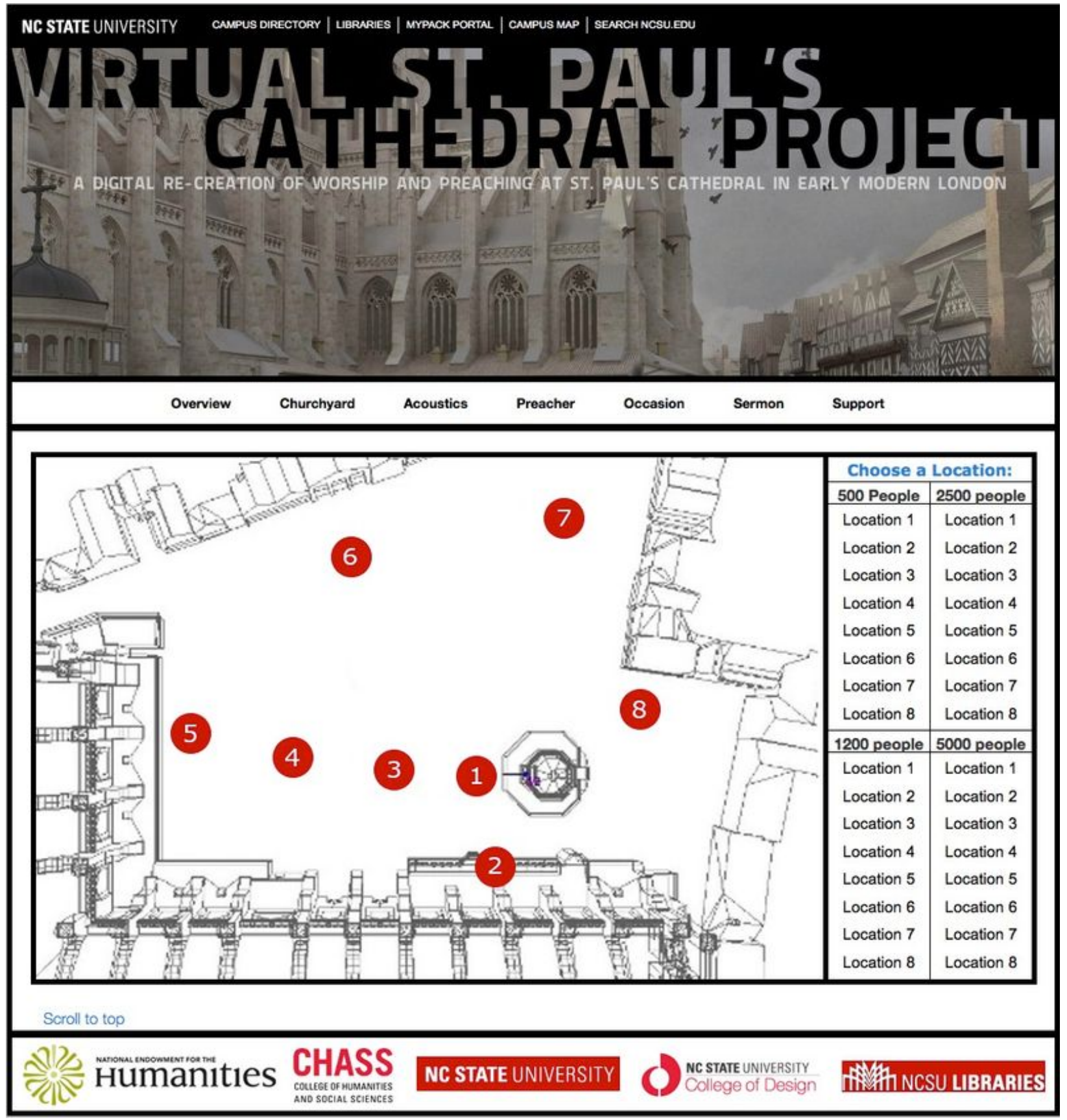

Figure 2. Virtual St. Paul's Cathedral Project, https://vpcp.chass.ncsu.edu/

Comme je l'expliquais au début de cet article, imaginer une performance des registres de la Comédie-Française revient à imaginer une série de scènes fugaces, à la manière d'un film accéléré qui mettrait en avant par son montage les discontinuités et qui se concentrerait sur la salle du théâtre. L'événement théâtral apparaitrait ainsi comme créé par les comédiens, les spectateurs et les objets matériels. Ces données pourraient- 
elles être utilisées pour élaborer un modèle qui respecterait ce point de vue, tout en acceptant les pauses du montage et les lacunes manifestes dans les informations ?

Les registres sont fascinants précisément parce que le chemin qu'il trace dans le matériau historique est absolument différent de celui qu'empruntent des projets spéculatifs, hypothétiques ou génératifs. Par exemple, ils présentent 1036 pièces, bien au-delà des quelques 200 œuvres constituant le répertoire sur lequel les spécialistes d’histoire littéraire s'appuient en général. Si les pièces célèbres du XVIII ${ }^{\mathrm{e}}$ siècle reviennent très souvent, elles côtoient des œuvres inconnues et oubliées, des succès sans lendemain, des vestiges du XVII ${ }^{\mathrm{e}}$ siècle et d'autres textes qui apparaissent comme des intrus sur la scène dominante des Lumières. L'architecture de l'interface numérique du Projet $\underline{\mathrm{RCF}}$ déconstruit la notion de chef-d'œuvre. Son interface de recherche fait apparaître l'habitude qu'avait la Comédie-Française de représenter deux pièces par soirée, grâce à une disposition qui met visuellement en avant ce jumelage et qui incite les historiens à envisager ensemble les deux textes. Bien que la vente et le prix des billets aient été les premiers éléments à retenir l'attention des chercheurs sur ces registres, il s'est avéré difficile de les interpréter et d'en donner des figurations graphiques qui en proposent une explication immédiate et fiable : les quatre théâtres différents et, au sein de chacun d'entre eux, les changements de configuration des sièges ne sont que quelques uns des obstacles qui rendent difficile la reconstruction de l'histoire de la Comédie-Française à partir de ses registres. Les données qu'ils contiennent réclament en effet une méthodologie adaptée aussi bien aux petites histoires, localisées et diverses, qu'ils racontent qu'à l'intuition qu'elles suscitent, à savoir qu'elles donnent accès à une expérience plus large, voire plus unifiée. Quel type de modèle est susceptible à la fois de traiter un ensemble de données concernant plus de trente-quatre mille représentations et de rendre compte d'un phénomène qui s'est répété durant plus d'un siècle dans quatre bâtiments différents ? Plutôt que des reconstitutions, comment tenter des expérimentations et des explorations qui élargissent le champ de ce que nous pensons pouvoir connaître du théâtre du XVIII ${ }^{\mathrm{e}}$ siècle ?

\section{Développer un modèle ouvert à l'ambiguïté : les écueils à éviter}

Une approche numérique-virtuelle d'archives historiques comme celles de la ComédieFrançaise se doit donc de suivre un modèle qui ne vise pas la reconstitution mais la variabilité et l'ambiguïté. Peut-être est-ce à cette condition que nous parviendrons à 
une histoire du théâtre qui évite l'écueil de célébrer les anomalies et les cas particuliers, comme celui de prétendre parvenir à des révélations qui ne font que dissimuler des tentations positivistes. La tentation d'une interprétation quantitative, qui semble aller naturellement de pair avec les enquêtes partant d'une base de données, accentue le danger de ces trois miroirs aux alouettes : l'illusion quantitative, l’illusion de la « découverte » et l'illusion téléologique.

La première de ces illusions consiste à appréhender ce qui sort de l'ordinaire à un niveau quantitatif comme étant le meilleur (en termes de succès, de revenus, d'affluence, d'importance), comme si l’on savait ce qui était considéré comme « le meilleur » durant la période considérée. Ainsi, les données risquent de nous faire croire que nous avons accès à une représentation complète et intelligible de ce qu'était un succès ou un échec. Bien entendu, certains cas particuliers, que ce soit celui d'une pièce, à l'instar du Mariage de Figaro, ou d'un auteur, comme Shakespeare, permettent de saisir un phénomène unique et donc intéressant. Ils ont toutefois aussi tendance à renforcer, délibérément ou non, nos catégories axiologiques $\underline{42}$. Au lieu de cela, s'intéresser à ce qui ne signale pas un succès quantitatif pourrait permettre de saisir d'autres déterminations du paysage sonore du théâtre. S'il va de soi que tout modèle d'interprétation doit excéder les indicateurs économiques, il doit aussi être conçu pour s'adapter à d'autres variables. Si nous devions étudier une pièce, il ne s'agirait donc pas d'en choisir une qui sort de l'ordinaire mais plutôt de s'intéresser à une œuvre qui partage certaines caractéristiques avec d'autres.

Le deuxième écueil, l'illusion de la « découverte », revient à essayer de reconstituer ou de découvrir la vérité historique grâce à la performance. Quel que soit le modèle, on a tendance à espérer qu'il donne une représentation du passé qui l'explique parfaitement et le rende accessible. C'est ce que montre J. Ravel dans son étude sur diverses études imprimées des registres ayant précédé le Projet $\underline{\mathrm{RCF}}$. Il explique que non seulement un modèle ne fait rien de tel mais que bien au contraire, il propose comme le propose Jeffrey Peters - quelque chose de tout à fait nouveau $\underline{43}$. De même qu'il se doit d'éviter l'illusion quantitative en s'intéressant à des objets ne sortant pas de l'ordinaire, un modèle visant à approfondir notre compréhension du passé se doit de ne pas s'appuyer, pour proposer une interprétation, sur le compte rendu unique d'un moment ou d'une situation, mais de privilégier une conception dynamique des questions historiographiques. Un modèle acoustique modulable ouvre de nouvelles voies pour s'interroger sur la représentation et le pouvoir des réseaux sociaux. Le but 
n'est pas de reconstituer une expérience, mais plutôt de comprendre l'étendue des possibles mis à disposition par l'archive.

Enfin, le dernier écueil consiste à vouloir confirmer ou réfuter des modèles téléologiques. Comme nous l'avons déjà dit, nous avons tendance à retrouver dans les données ce que nous connaissons déjà. Comment confirment-elles les évolutions du théâtre des Lumières ? Ou, de manière plus caricaturale, comment peuvent-elles annoncer la Révolution ? C'est pourquoi nous avons besoin d'un modèle qui vise non pas à faire voir le passé mais à nous aider à interroger l'archive. De nouveau, puisqu'il ne s'agit pas de reconstituer une expérience, ce qui serait impossible de toute manière, le but est de renouveler nos approches de ces données et de les présenter autrement afin de leur poser des questions différentes. Un modèle acoustique modulable pourrait être un exemple, non pas d'une tentative de reconstituer le passé, mais de se confronter aux questions que celui-ci nous amène à nous poser.

Même la question de l'acoustique, en tant que porte d'entrée idéale pour concevoir la représentation comme un événement incarné et construit en réseaux, doit être modélisée à plus petite échelle. C'est là que son caractère modulable entre en ligne de compte. Pouvons-nous utiliser un modèle pour explorer différents scénarios ? Par exemple, la relation entre le nombre de billets vendus et celui des spectateurs assistant à une représentation donnée constitue l'un des points sur lequel achoppent les chercheurs s'intéressant à la Comédie-Française. Christophe Schuwey et Christopher Morse se sont attaqués à cette question lors du colloque international du Projet RCF de mai 2016 (Harvard-MIT). Ils ont créé pour la saison 1784-1785 une visualisation qui « révèle l'affluence lors d'une représentation donnée sous la forme d'une carte thermique : plus la couleur est chaude, plus la représentation a attiré de spectateurs ». Non seulement cette expérience parvient à son but - faire apparaître visuellement la densité du public au fil de la saison - et rend compte précisément de la façon dont les recettes évoluent pendant cette période. Mais surtout, elle permet de repérer des erreurs potentielles dans les données. C. Schuwey et C. Morse ont identifié une anomalie : la séance du 27 avril 1784. La carte thermique correspondant à ce jourlà représente la troisième rangée en bleu, indiquant ainsi qu'elle était vide. C'est historiquement improbable dans la mesure où il s'agit de la première très attendue du Mariage de Figaro. « Est-ce qu'il pourrait s'agir d'une erreur dans les données, ou estce lié à une disposition différente des sièges ou encore aux entrées achetées à l'avance pour la saison ? À l'aide de ce guide présentant visuellement chaque représentation, il est beaucoup plus facile d'identifier ces incohérences et de mener l'enquête $\underline{44}$ ». C. Schuwey et C. Morse sont en train d'étendre leur projet aux 113 saisons couvertes 
par les registres et d'inclure des graphiques spécifiques aux quatre salles où la troupe de la Comédie-Française a joué.

Pourrions-nous aussi utiliser les registres pour déterminer l'ambiance sonore caractéristique d'une représentation ayant attiré peu de spectateurs, par rapport à celle que produirait la représentation d'une même pièce avec une forte affluence, et pour comprendre les conséquences de ces bruits sur le déroulement du spectacle dans l'un et l'autre cas ? Serait-il possible d'ajouter à ce modèle la manière dont les sons produits par les spectateurs se propagent et transforment l'expérience ? Dans quelle mesure les loges onéreuses auxquelles J. Clarke fait référence permettaient-elles de voir et d'entendre la pièce, mais aussi d'être vu et donc d'être entendu ? En outre, ce modèle pourrait être utile pour saisir les transformations importantes de la représentation au cours du XVIII ${ }^{\mathrm{e}}$ siècle. Quelle a été, par exemple, la conséquence acoustique de la disparition des spectateurs sur scène après 1759 , que ce soit pour les comédiens ou pour le paysage sonore, altéré par ces changements dans l'agencement des places ? Dans quelle mesure le souci de Voltaire pour l'illusion théâtrale a-t-il été influencé par ce qu'il observait chez les spectateurs sur scène et par ce qu'il a pu entendre?

J'en reviens au VPCP de J. Wall et à ce qui m'a le plus intriguée : l'apport pour la recherche de la modélisation du sermon. Deux éléments dans ce processus semblent particulièrement importants. Le premier est lié ce que J. Wall a appris au sujet de la performance orale d'une œuvre de ce genre. Le discours de Donne a été écrit pour être prononcé en extérieur, à proximité de murs en pierres et de la façade de l'église, qui auraient dû créer un écho, et à proximité également des structures en bois des maisons qui, elles, auraient absorbé les sons. Dans ces conditions, la taille de la foule aurait eu des conséquences importantes sur la qualité du son et sur sa portée : combien de personnes auraient pu entendre Donne, à quelle distance et avec quelle précision ? J. Wall et son équipe se sont aperçus qu'à cause des réverbérations sonores, Donne avait sans doute prévu d'énoncer son discours lentement pour éviter que l'écho couvre ses paroles. Qu'est-ce que la modélisation de la dimension orale d'une performance pourrait nous apprendre sur le paysage sonore du théâtre ? Il faudrait tenir compte des intensités respectives des phonèmes en français, comme l'a fait Bruce Smith pour le théâtre anglais, et des emprunts que les comédiens cherchant à se faire entendre firent à l'art oratoire, aussi bien civil que religieux. Je pense en particulier aux travaux de Pierre-Alain Clerc sur le débit, c'est-à-dire la vitesse d'énonciation des alexandrins. Un modèle acoustique se concentrant sur une pièce en particulier représentée tout au long du siècle pourrait nous permettre de préciser les 
types de changements qu'aurait connus, selon P.-A. Clerc, l'art oratoire au fil du temps, mais aussi l'art du comédien, comme l'a étudié Sabine Chaouche $\underline{45}$.

La seconde conclusion que je veux emprunter au projet de J. Wall est la façon dont il a placé au premier plan la participation des fidèles - la foule - pour comprendre la signification historique de la prédication et de la persuasion religieuse. Dans le modèle qu'il propose, cette foule apparaît comme un pivot pour concevoir le sermon comme un événement. Le VPCP confirme ainsi l'idée défendue par le sociologue Bruce Reed dans The Dynamics of Religion et que J. Wall explique en ces termes : la pratique de la foi religieuse est « une disposition, une orientation physique et intellectuelle, dont les contours se dessinent au fil de participations à différents événements, qui établissent et nourrissent une compréhension de soi en terme de réseaux sociaux et de participation commune à l'établissement de relations $\underline{46}$ ». Est-ce que la modélisation du public rendue possible à partir des registres pourrait nous permettre d'en savoir davantage sur le théâtre conçu comme un ensemble de pratiques par lesquelles un individu se construit et développe ses croyances au sein de réseaux sociaux ? Ne pourrait-on pas identifier un pouvoir de la foule, celui d'influencer la représentation par sa présence - un pouvoir qui serait à concevoir non pas en termes financiers, mais en termes proprement économiques, comme capacité à transformer les énergies et les ressources?

\section{Une pièce idoine : le cas d'Athalie}

Comment, donc, concevoir un modèle acoustique modulable fondé sur les données des registres ? Il faut prendre en compte les informations sur les pratiques scéniques contenues dans les traités sur l'art dramatique, la musique et le chant; il faut également considérer les indications sur l'architecture des bâtiments et sur leurs matériaux, sur l’histoire des décors, ainsi que les témoignages de comédiens, de spectateurs, d'auteurs, et enfin des éléments présents dans les dossiers de police et dans des mémoires. C'est dans cet ensemble acoustique élargi que les données numériques du Projet $\underline{\mathrm{RCF}}$ trouveraient leur place.

Le millier de pièces, les quatre lieux de représentations qui furent plusieurs fois transformés, les modifications dans le calendrier du théâtre et les événements historiques externes ayant pu influencer la programmation sont autant de variables, parmi d'autres, qui rendent presque impossible une approche qui chercherait à recomposer un espace physique miniature : quel lieu et quelle période choisir ? Étant 
donné l'étendue des données et leur hétérogénéité, il faudrait d'abord identifier un certain nombre de paramètres dont la stabilité relative serait à même de structurer le modèle. On est ainsi tenté de sélectionner un théâtre, une saison ou une pièce. Lors de ce choix, je crois qu'il serait crucial de développer un modèle qui fasse de la variabilité et de l'ambiguïté ses principes, un modèle qui préserve et cultive la nature du théâtre comme quasi-objet. Ici aussi, je pense qu'il est possible de s'inspirer du VPCP, dont la source originelle est traversée de tensions : un sermon oral mais immédiatement transcrit, improvisé mais aussi répété, pensé pour un parvis mais finalement prononcé dans la cathédrale. En d'autres termes, le but ne devrait pas être de reconstituer un événement ou de saisir l'histoire mais plutôt d'aspirer à un modèle qui s'adapterait à une diversité de questionnements, concernant aussi bien la représentation théâtrale elle-même que la nature des données sur lesquelles le modèle est construit.

Un tel modèle acoustique modulable, rendant compte d'une pièce définie comme l'événement créé par la rencontre entre des acteurs, un espace et un public, constituerait un laboratoire pour explorer notre compréhension du théâtre du XVIII ${ }^{\mathrm{e}}$ siècle. Si l'on enregistrait la lecture d'un texte suivant ce modèle, l'enregistrement devrait être modifié en fonction de l'acoustique, c'est-à-dire selon ce que nous savons de l'architecture du théâtre et de ses matériaux, des transformations de sa disposition et des variations d'affluence d'une représentation à l'autre. La pièce qui servirait de cas d'étude ne devrait toutefois pas être choisie à partir d'une quelconque singularité quantitative. En effet, sélectionner ce que nous considérerions comme le plus grand succès du temps (que ce soit en termes de fréquence dans la programmation, ou en termes d'importance esthétique ou politique) serait une erreur : nous ne savons pas ce qu'est « le meilleur » pour l'époque, et cela reviendrait donc à traiter les données comme une représentation transparente d'un succès ou d'un échec. Si l'on voulait enregistrer le texte d'une pièce, il faudrait donc en choisir une qui n'a pas été un succès quantitatif ou qui ne constitue pas une exception. Au lieu de cela, choisir une pièce qui a duré sans faire de coup d'éclat, qui a été jouée régulièrement, fût-ce sans grandes conséquences, permettrait sans doute de faire apparaître les autres variables qui influent sur le paysage sonore du théâtre.

De ce point de vue, il apparaît légitime de laisser de côté la pièce la plus populaire du siècle, le Mariage de Figaro de Beaumarchais (1784), la plus révolutionnaire, Charles $I X$ de Chénier (1789), ou l'une des «premières », comme Zaïre de Voltaire, la première tragédie « française » (1732), pour élire une pièce à l'opposé de tout cela : un vestige du XVII ${ }^{\mathrm{e}}$ siècle, Athalie de Racine (1691). Au début de sa carrière, elle n'a été représentée que trois fois, pour des membres de la cour de Louis XIV, avant que sa 
représentation publique soit interdite pendant plusieurs décennies. Ainsi, la dernière pièce de Racine semble de prime abord atypique : elle est le produit d'une cour vieillissante et des liens que celle-ci entretenait avec les jeunes filles nobles de l'école de Saint-Cyr, et sa genèse ainsi que ses débuts la distinguent des pièces au répertoire de la Comédie-Française au XVII ${ }^{\mathrm{e}}$ siècle. Néanmoins, les singularités d'Athalie - ou à certains égards sa banalité - ne sont pas plus importantes pour mon propos que son entrée à la Comédie-Française et que sa présence continue au répertoire tout au long du XVIII ${ }^{\mathrm{e}}$ siècle.

Athalie fut jouée pour la première fois le 5 janvier 1691, puis de nouveau le 8 février, par les jeunes filles de la Maison royale de Saint-Louis dirigée par Mme de Maintenon à Saint-Cyr, pour quelques spectateurs choisis de la noblesse, et une dernière fois le 22 février pour la Reine et le Roi d'Angleterre alors en exil à Saint-Germain. Interdite de représentation publique, elle fut cependant jouée en privé. Il faut attendre 1702 pour qu'Athalie réapparaisse, lors du carnaval, à l'occasion d'une représentation pour le roi. Elle n'est cependant pas jouée par des acteurs professionnels, mais par des membres de la cour, dirigés par Michel Baron, « le vieux Baron », de la troupe de Molière. Selon Georges Forestier, « on garde la trace d'une représentation privée qui eut lieu en 1714 chez la duchesse du Maine [...]. Pour la première fois des acteurs professionnels tinrent les rôles principaux. Et l'on est en droit de penser que ce ne fut pas un cas isolé $\underline{47}$ ». L'interdiction fut officiellement levée en 1716 par le duc d'Orléans, qui avait lui-même joué dans le spectacle de 1702, sans le chœur. G. Forestier affirme que la pièce a ensuite été donnée quatorze fois, quand la base de données du Projet RCF ne garde la trace que de treize représentations.

\section{Visit the web version of this article to view interactive content.}

\section{Figure 3. Source : Outil de découverte, Projet RCF.}

La pièce fut jouée 229 fois au cours du XVIII ${ }^{\mathrm{e}}$ siècle selon G. Forestier et à 206 reprises selon le Projet RCF. Ces chiffres sont remarquables en cela qu'ils ne font pas particulièrement ressortir Athalie dans l'ensemble des pièces de Racine. Phèdre fut jouée 424 fois, Andromaque 296 fois, et Britannicus 289 fois. Athalie a été jouée davantage que Bajazet (184), Bérénice (78), Esther ou La Thébaïde (8 fois chacune), sans mentionner Alexandre le Grand (3). L'histoire de ses représentations se poursuit au siècle suivant, durant lequel elle est même donnée davantage, à 255 reprises selon 
G. Forestier. L'intérêt particulier de ces données ne réside pas dans la légère augmentation quantitative, ni dans une popularité accrue : toujours selon G. Forestier, elle passe du sixième rang au cinquième rang en termes de popularité, bien devant Mithridate. Ces données sont intéressantes parce qu'elles indiquent un intérêt continu pour la pièce : Athalie se maintient. Une relique de l'austérité paradoxalement spectaculaire du règne de Louis XIV a survécu tout au long du XVIII ${ }^{\mathrm{e}}$ siècle, tant et si bien qu'elle devient, selon Renaud Bret-Vitoz, « davantage une tragédie du $\mathrm{XVIII}^{\mathrm{e}}$ siècle que du XVII ${ }^{\mathrm{e}}$ siècle $\underline{48}$ ».

G. Forestier ne compare Athalie qu'aux autres pièces de Racine. Dans le contexte de la Comédie-Française, la question de l'impact doit être envisagée différemment. Derek Miller a retracé le devenir du répertoire de 1680 et la carrière des pièces jouées cette saison-là tout au long du XVIII ${ }^{\mathrm{e}}$ siècle $\underline{49}$. Il observe avec sagacité comment l'utilisation de données chiffrées brutes avantage mécaniquement les pièces les plus anciennes. À la place, il organise sa liste en fonction du nombre de représentations par année pour les pièces jouées sur plus de cinq saisons, ce qui lui permet de faire ressortir la popularité persistante du Mariage de Figaro tout au long du siècle ainsi que l'importance d'un répertoire de base représenté de manière continue, même sans succès éclatants. Athalie fait partie de cet ensemble de pièces durables. Jouée pour la première fois en 1715, elle a été représentée chaque décennie sur plus de cinquantecinq saisons jusqu'en 1791. La période la plus longue durant laquelle la tragédie n’a pas été jouée n’a duré que cinq ans, de 1733 à $1738 \underline{50}$.

De la sorte, on sait qu'Athalie fut jouée dans les trois salles occupées par la ComédieFrançaise au XVIII ${ }^{\mathrm{e}}$ siècle. Il est intéressant d'imaginer un modèle acoustique qui se distinguerait des réalités virtuelles immersives et qui pourrait s'adapter aux différents bâtiments où la troupe s'installa. Privilégier le son, au détriment d'une entreprise de reconstitution de la salle de chaque théâtre, permettrait de suivre la fortune mouvante de la Comédie-Française d'un lieu à l'autre, en se fondant pour comprendre ces changements sur les transformations sonores, et non sur des extrapolations de ce que l'on y voyait.

Compte tenu de ses représentations, on peut considérer qu'Athalie est l'exemple d'une pièce dont le succès a diminué au fil des ans : elle n'a jamais été autant jouée que durant les premières années de son entrée au répertoire et n’a parfois été donnée qu'une seule fois sur une saison (1725-26, 1741-42, 1747-48, 1778-79, 1780-81, 178283). Néanmoins, elle fut représentée entre vingt et trente fois par décennie, à l'exception des années 1730, où elle disparaît de la scène pour une grande partie de la 
période. On peut en déduire que la tragédie constitue un cas d'étude satisfaisant pour une interprétation sur le long terme de la carrière d'une pièce n'ayant rien d'exceptionnel pour la Comédie-Française.

Dire qu'elle n'a rien d'exceptionnel est pour moi une manière de marquer la différence entre la réception du public et celle de la critique actuelle. Aujourd'hui, étant donné notre compréhension des genres, de la carrière de Racine et de la fortune de ses pièces au XVIII ${ }^{\mathrm{e}}$ siècle et au-delà, on estime qu'Athalie est bel et bien exceptionnelle. Mais ce n'est pas le cas si l'on regarde la réception dont les registres rendent compte. Les recettes vont ainsi de 276 livres (le 27 mars 1722, où la tragédie est jouée avec la comédie de Du Fresny L'Esprit de contradiction) à 5 708,80 livres (le $1^{\mathrm{er}}$ août 1788, où elle est jouée avec Le Bienfait anonyme de Joseph Pilhes). Athalie offre donc un éventail allant de la médiocrité au succès et peut ainsi être considérée comme représentative de tous les cas «typiques » que l'on trouve dans les registres pour le $\mathrm{XVIII}^{\mathrm{e}}$ siècle. Laffirmation est radicale, et contredit les évaluations littéraires et historiques de Racine et de cette pièce en particulier. En effet, la critique présente généralement Athalie comme une anomalie à bien des égards - que ce soit au sein de l'œuvre racinienne, parmi les tragédies de la première modernité jouée du temps de Louis XIV, parmi le théâtre à machines ou encore parmi les pièces religieuses. Cette interprétation provient souvent du fait qu'il s'agit de sa seule tragédie complète tirée des Écritures et du caractère exceptionnel de la représentation de la violence sur scène.

Si on lit les critiques du XVIII ${ }^{\mathrm{e}}$ siècle, il semble que le statut d'Athalie ait été pour le moins ambivalent. De manière générale, les tragédies de Racine sont reconnues pour leurs qualités poétiques mais considérées comme des poèmes manquant de théâtralité. Bien qu'Athalie soit louée pour la beauté de ses vers par Voltaire, d'Alembert et Marmontel, entre autres, elle est aussi critiquée pour l'invraisemblance de son action et de ses personnages $\underline{51}$. Certains voient dans les scènes finales, dont la dimension spectaculaire est surprenante pour une pièce de Racine, un modèle de ce « pathétique » qui était alors célébré. Athalie est ainsi intéressante dans l'histoire de la Comédie-Française dans la mesure où elle fut à la fois rejetée et imitée, et où elle influença les pratiques théâtrales du XVIII ${ }^{\mathrm{e}}$ siècle. 


\section{Les échos d'Athalie sur la scène des Lumières}

Malgré les critiques du XVIII ${ }^{\mathrm{e}}$ siècle qui condamnent plus ou moins explicitement les formes tragiques antérieures, Athalie joue néanmoins un rôle important, parfois paradoxal, dans les écrits sur l'art dramatique de l'époque. À cet égard, sa dimension sonore est essentielle. Multiple, intégrée à la composition et à la structure de la pièce, cette sonorité, parfois louée et parfois décriée, constitue un élément crucial dans la réception critique et l’histoire de ses représentations.

Tout d'abord, témoin de la promotion par la critique du XVIII ${ }^{\mathrm{e}}$ siècle d'un théâtre spectaculaire faisant la part belle à la vision et aux sens, Athalie joua un double rôle. La qualité poétique reconnue de ses vers en fit une source privilégiée pour imaginer le monde théâtral sous Louis XIV, comme en témoigne le tableau du début du XIX ${ }^{\mathrm{e}}$ siècle peint par Julie Philipaut et représentant Racine récitant sa pièce [Fig. 4]. Le dramaturge n'y apparaît pas en train de jouer mais en train de déclamer. Les mains de Madame de Maintenon et celles du roi sont également saisies alors qu'elles imitent les gestes du récitant. Le tableau synthétise la double vision que l’on se fait, au $\mathrm{XVIII}^{\mathrm{e}}$ siècle et au siècle suivant, de la tragédie du XVII ${ }^{\mathrm{e}}$ siècle : elle est vue à la fois comme une pratique figée, voire comme la relique guindée d'un régime politique obsolète, et comme un exemple captivant de poésie mettant en branle les émotions des spectateurs. 


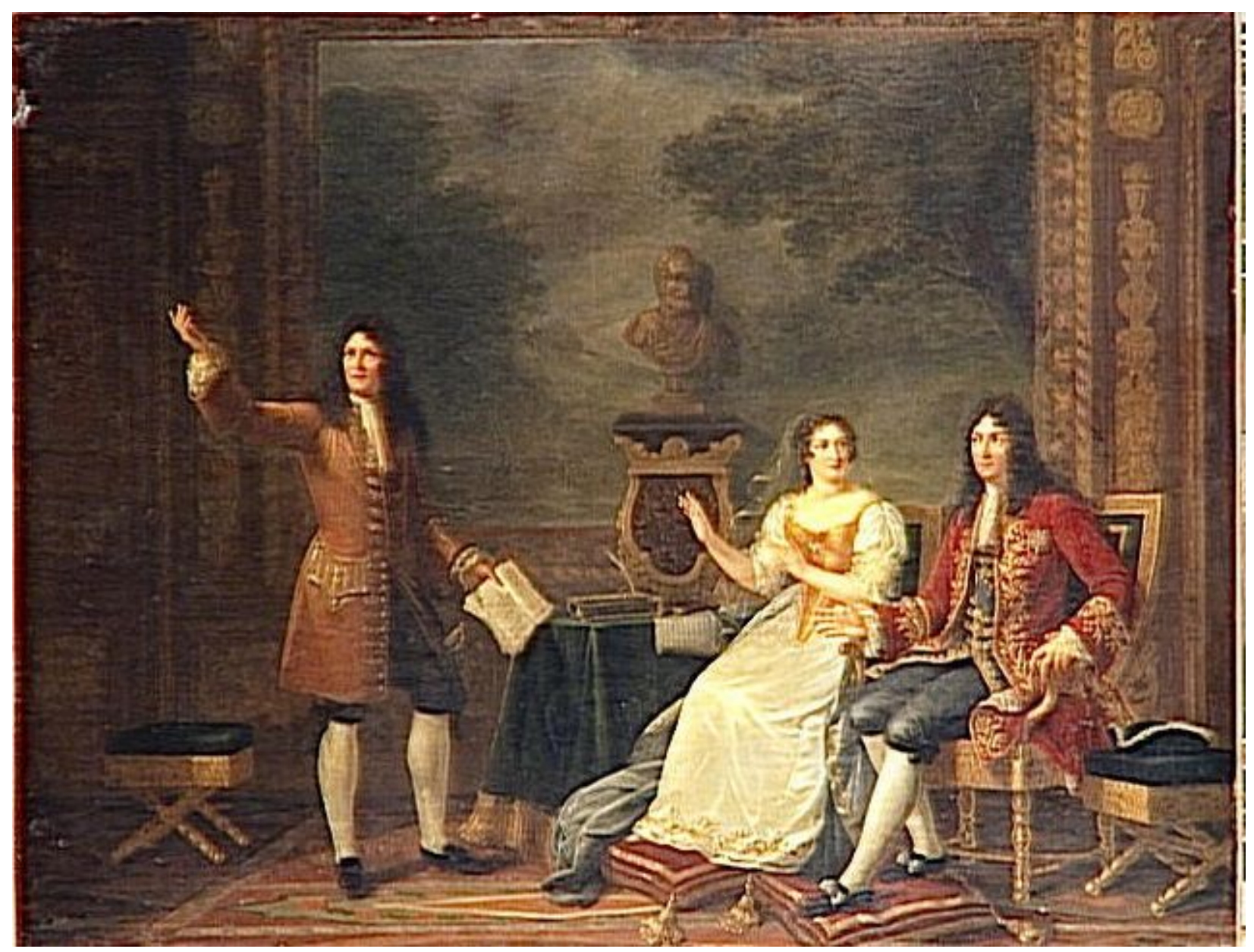

Figure 4. Julie Philipaut, Racine lisant Athalie devant Louis XIV et Madame de Maintenon, 1819.

Même s'il est évident que la transcription acoustique d'une pièce permettrait de rendre compte de l'évolution de la déclamation et du jeu, Athalie est particulièrement intéressante par sa trajectoire : elle devint au cours du XVIII ${ }^{\mathrm{e}}$ siècle à la fois un exemple d'excellence poétique et une source de spectacle. Si la représentation que propose Philipaut des débuts de la pièce célèbre l'un des deux pans de cet héritage, l'autre est tout aussi important.

Au fil du XVIII ${ }^{\mathrm{e}}$ siècle, le rôle-titre d'Athalie fut joué à plusieurs reprises par des comédiennes de renom : grâce aux registres, on connaît l'interprète de l'héroïne éponyme pour chaque représentation. Des sources extérieures, comme Le Mercure galant, permettent de confirmer non seulement la distribution mais aussi la réception des différentes interprétations $\underline{52}$. Est-ce qu'un enregistrement d'Athalie incluant des variables modulables nous permettrait de reconsidérer ce que l'on sait des différents 
styles de jeu de Desmares, de Dumesnil, de Duclos, de Lecouvreur et de Clairon, ainsi que de la manière dont ils étaient perçus ? [Fig. 5] En irait-il de même pour Baron, qui interpréta avec grand succès Joad en 1721 lors de son retour sur scène, ou pour les enfants qui jouaient Joas?

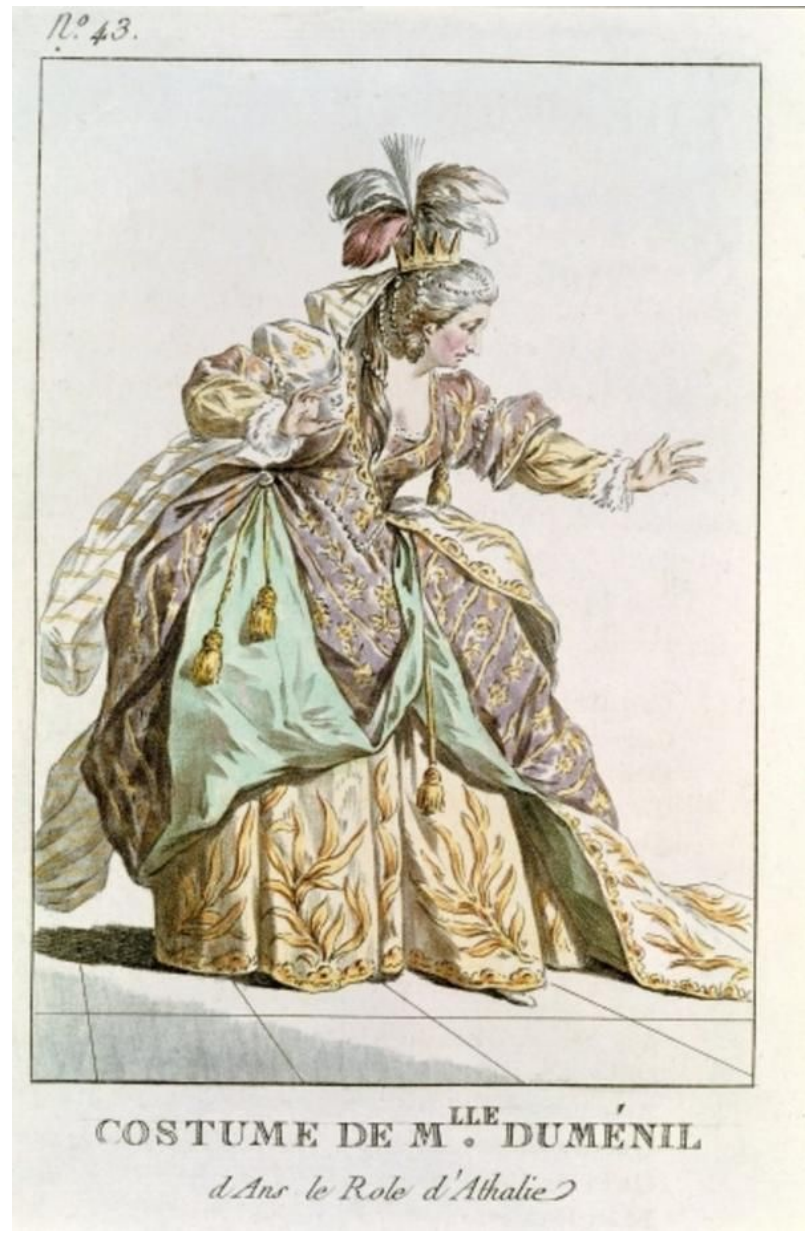

Figure 5. Costume de Mlle Dumesnil dans le rôle d'Athalie, reproduit avec la gracieuse autorisation de la Bibliothèque-Musée de la ComédieFrançaise.
Les critiques de l'époque comparaient les interprétations. Un modèle acoustique modulable pourrait permettre de mieux saisir l'esthétique de l'interprétation dramatique et les polémiques que soulevèrent les différents styles de jeu.

Athalie avait été écrite avec musique et chœurs, avec une partition de JeanBaptiste Moreau. De manière remarquable cependant, la tragédie ne fut représentée que huit fois avec de la musique. De plus, la première représentation en musique n'eut lieu ni l'année de la création en 1691, ni avec la musique de Moreau, mais plus tard, avec une partition de François-Joseph Gossec et des solos de Haydn. La seule représentation d'Athalie accompagnée de la musique de Moreau se déroula en 1770 à l'Opéra de Versailles, avec quelques ajouts empruntés à d'autres œuvres $\underline{53}$. Une modélisation acoustique d'Athalie pourrait inclure ces variables : ce serait l'enregistrement d'une performance qui n’a jamais eu lieu, suivant la partition de Moreau pour le chœur, avec les variations introduites par les autres morceaux joués par la suite. On pourrait aussi proposer un scénario contrefactuel en utilisant la partition de Moreau et se demander si la musique originale constituait en elle-même un problème pour les spectateurs. En effet, on sait qu'Athalie, peut-être comme d'autres pièces accompagnées de musique, a connu des ajustements : la partie chantée fut parfois 
raccourcie, voire coupée. Le Mercure galant des mois de juin et juillet 1721 signale que lors de la représentation de la tragédie cette saison-là « les Comediens en ont retranché la plus grande partie des Chœurs \& tout le chant » [Fig. 6]. Le paysage sonore d'Athalie est ainsi très riche : aussi bien la présence que l'absence des chœurs, de la musique et des différents interprètes détermine son archive sonore potentielle.

Qu'Athalie soit surtout louée en tant que pièce à machines donne à comprendre la manière dont la dimension scénique de la représentation a pu influencer l'expérience théâtrale. La révélation que Joas est le roi légitime a lieu dans les scènes 4 et 5 de l'acte $\mathrm{V}$ : une machine ouvre le décor en fond de scène et révèle un sanctuaire caché dans le temple, d'où émerge le jeune souverain, couronné sur son trône et entouré de soldats qui entraînent Athalie dans la coulisse pour la mettre à mort. Ainsi que l'a expliqué R. BretVitoz, c'est grâce à ses machines qu'Athatlie est devenue « le parangon de la tragédie sublime et spectaculaire qui rompt avec l'esthétique antécédente $\underline{54}$ ». La mise en scène spectaculaire de la révélation est unique dans l'œuvre de Racine et nécessitait assurément l'utilisation de machines quand elle était jouée à la Comédie-Française. La technologie des machines va de pair avec un type de sons particulier. Dans quelle mesure le grincement des poulies ou le roulement des roues sur leurs rails participaient à la cacophonie de la scène ? Comment cela affectait-il la déclamation des

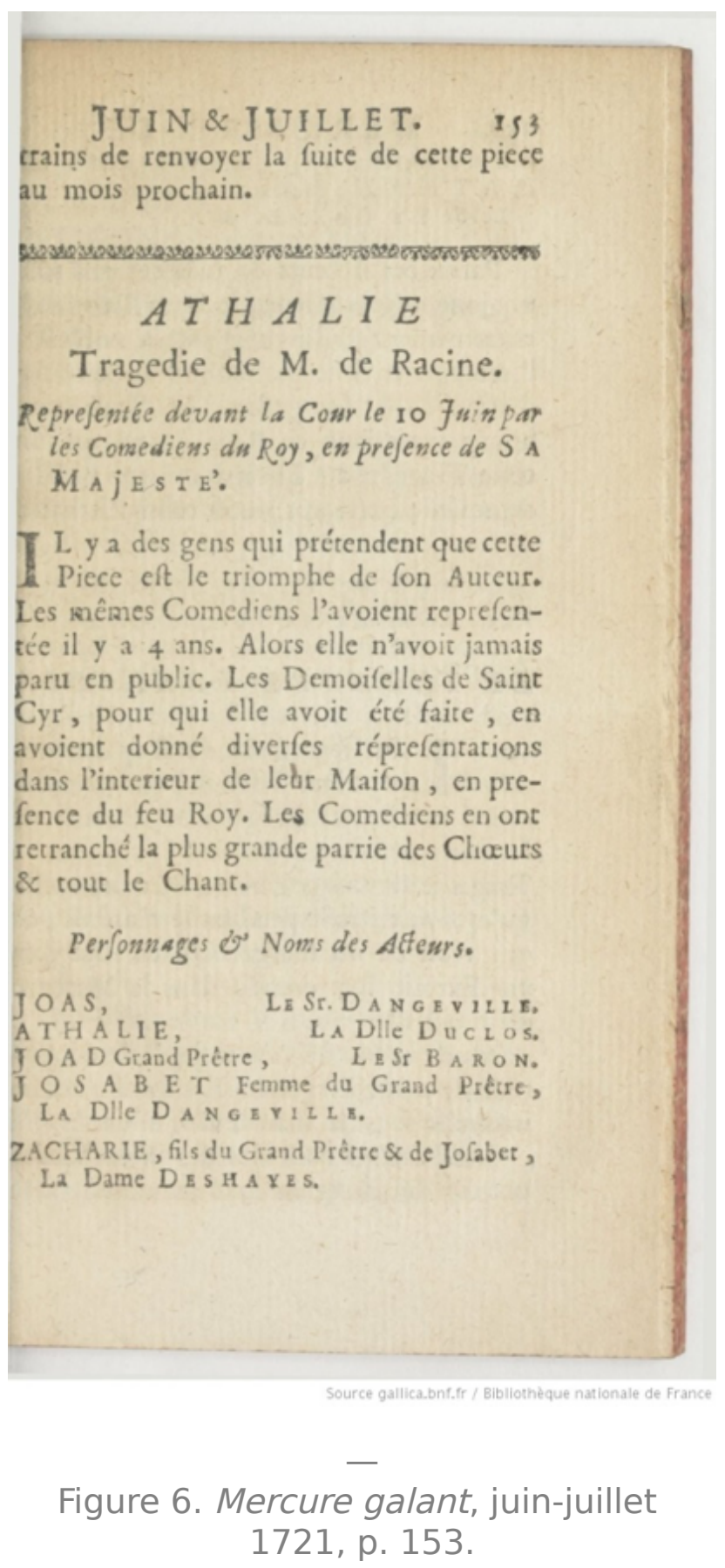
1721, p. 153. 
vers ? Utilisait-on à un niveau dramaturgique l'interaction entre le son des machines et la voix des acteurs pour avancer vers le tableau final ?

De manière tout aussi importante, un modèle acoustique modulable nous permettrait de jouer avec des sons qui perturbent la représentation : quelles étaient les conséquences des huées du public ? Quels étaient les sons produits quand le parterre jetait des oranges sur un noble arrogant dont le siège leur bloquait la vue ? Combien de personnes étaient nécessaires pour produire un tintamarre qui rende une pièce inaudible et ainsi faire passer plus rapidement à la seconde ? Un modèle acoustique modulable nous inviterait à traquer et à écouter les anomalies qui donnaient au théâtre sa capacité non pas seulement de refléter la société, non pas seulement de la troubler, mais aussi de la changer.

\section{Conclusion. Que les incertitudes du passé nous réduisent au silence}

Comme W. Ernst, je suis convaincue que les archives peuvent avoir un son. Ma proposition de composer, à partir des données du Projet $\underline{\mathrm{RCF}}$ et d'autres informations contextuelles, un modèle acoustique modulable et de créer un enregistrement sonore ajustable d'une pièce particulière nous permettrait d'associer sources quantitatives et sources qualitatives. Aussi est-il possible d'aller au-delà de la reconstitution d'une ou plusieurs représentations passées, et d'essayer d'utiliser ce modèle acoustique multiforme, composé de différents acteurs, pour penser véritablement avec l'archive historique et laisser cette dernière nous poser des questions au lieu de « décider à l'avance à quoi doit ressembler l'ameublement du monde $\underline{55}$ », comme l'écrit B. Latour. Quelles incertitudes peut-on entendre dès lors que l'on accepte que les matériaux et les gens du passé interagissaient avec les lieux qu'ils occupaient?

De manière plus importante encore, je crois qu'un modèle acoustique modulable, grâce à ses capacités dynamiques d'adaptation, nous pousserait à abandonner les récits des origines auxquels nous continuons de nous accrocher. Autrement dit, en dessinant des questions à explorer, ce modèle pourrait nous réduire au silence de manière productive. En effet, on peut convenir que, bien souvent, les sons les plus audibles dans les archives proviennent des historiens (une quinte de toux, un soupir, un reniflement, un clic, un juron, un redémarrage d'ordinateur) qui font parler des données muettes en les interprétant. C'est ce que Michel Chion, dans son travail sur l'image cinématographique et les sons, appelle l'acousmêtre, cette voix invisible, qui semble se tenir juste en-dehors de l'écran, dont la force tient à ce qu'elle est hors 
cadre mais qu'elle menace à tout moment d'apparaître - une version acoustique du panoptique foucaldien, pour ainsi dire $\underline{56}$. En tant qu'acousmêtres des archives et de l'histoire que nous construisons, je pense qu'il nous faut, avant tout, faire attention à ne pas prétendre pouvoir un jour laisser parler les données par elles-mêmes. Nous sommes toujours présents; le premier son de l'archive est notre propre bruit $\underline{57}$.

\section{Footnotes}

1. Jeffrey S. Ravel, The Contested Parterre. Public Theater and French Political Culture, 1680-1791, Ithaca et Londres, Cornell UP, 1999, p. 54. NdT: Toutes les citations en langue originale anglaise figurant dans cet article ont été traduites par nos soins. $\bullet$

2. Pour une vue d'ensemble de l'histoire du son au théâtre, voir Ross Brown, Sound. A Reader in Theatre Practice, Londres et New York, Palgrave Macmillan, 2010.

3. «C'est donc à partir de ce fait, la contradiction, l’hétérogénéité, le champ de forces des instances, la multiplicité des points de vue, et en même temps leur présence éphémère et la présence éphémère d'une assemblée se divertissant et pensant, en un seul lieu et en un seul temps, qu'il faut bien réfléchir ; et s'interroger sur le fait qu'il y a alors, dans le présent éphémère de l'expérience théâtrale, dans la performance et dans la séance, une sorte de mise en place et de mise en jeu du commun, d'un jeu de l'individuel par rapport à ce commun: un phénomène de lien, et un jeu avec ce lien, à l'intérieur du lieu de spectacle et de son institutionnalisation en théâtre » (Christian Biet et Christophe Triau, « La comparution théâtrale. Pour une définition esthétique et politique de la séance », Tangence, $\left.\mathrm{n}^{\circ} 88,2008, \mathrm{p} .35\right)$.

4. Wolfgang Ernst, Sonic Time Machines. Explicit Sound, Sirenic Voices, and Implicit Sonicity, Amsterdam, Amsterdam UP, 2016, p. 101.

5. La tripartition du paysage sonore que propose R. Murray Schafer - c'est-àdire « ses notes dominantes, ses signaux et ses marques sonores »- permet de mettre en évidence la façon dont on laisse de côté ou au contraire privilégie certains des éléments sonores d'une culture. Tandis que les « notes dominantes » (ou sons ambiants) sont facilement éludées, et que les « signaux » se font généralement entendre selon la fonction spécifique qui leur a été donnée, les «marques sonores » sont ces repères acoustiques qui distinguent une 
communauté ou qui ont un sens spécifique pour elle, et qui pour cette raison «[méritent] d'être protégées » (The Soundscape. Our Sonic Environment and the Tuning of the World [1977], Rochester, Destiny Books, 1994, p. 9). 6. Wolfgang Ernst, « History or Resonance ? Techno-Sonic Tempor(e)alities », Journal of Visual Culture, $\mathrm{n}^{\circ} 14-1$, avril 2015, p. 104.

7. Peggy Phelan, Unmarked. The Politics of Performance, Londres et New York, Routledge, 1993, p. 146.

8. Wolfgang Ernst, art. cit., p. 104. L'usage assez libre que W. Ernst fait du mot « sonagramme » s'explique par la distinction comparative qu'il établit avec le sonogramme, au sein d'un débat plus large entre musicologues sur l'analyse et la représentation des sons. Pour une réflexion plus avancée sur les enjeux de la représentation visuelle du son, voir Stephen McAdams, Philippe Depalle et Eric Clarke, «Analyzing Musical Sound », dans Eric Clarke et Nicholas Cooke (dir.), Empirical Musicology, New York et Oxford, Oxford UP, 2004, p. 157-196. 9. Joan DeJean voit dans une édition paru à Amsterdam en 1683 du Festin de Pierre de Molière une version plus proche de l'original parce qu'elle a contourné la censure française en s'appuyant sur le script et sur les souvenirs d'un acteur. Voir son «Introduction » à Molière, Le Festin de Pierre (Dom Juan), éd. Joan DeJean, Genève, Droz, 1999, p. 22-23.

10. Jeffrey S. Ravel, op. cit., p. 131.

11. Ibid., p. 131.

12. Ibid., p. $220 . \Xi$

13. Voir Jan L. Clarke, "L'acoustique théâtrale au XVII ${ }^{\mathrm{e}}$ siècle : le cas de la Salle des Machines », dans Xavier Bisaro et Bénédicte Louvat-Molozay (dir.), Les Sons du théâtre. Angleterre et France (XVI ${ }^{e}-X V I I I^{e}$ siècle). Éléments d'une histoire de l'écoute, Rennes, Presses Universitaires de Rennes, 2013, p. 23-42 ; Pannill Camp, The First Frame. Theatre Space in Enlightenment France, Londres, Cambridge UP, 2014.

14. Pierre Frantz, L’Esthétique du tableau dans le théâtre du XVIII siècle, Paris, Presses Universitaires de France, 1998.

15. Pannill Camp, op. cit., p. 151-152. 
16. Ibid., p. 160-163.

17. Agathe Sanjuan, «Mesures de la fréquentation du public dans la salle : quelques considérations », site RCF, https://www.cfregisters.org/fr/espaceencyclopédique/parcours-des-registres-journaliers/parcours-des-registres-imesures-de-la-fréquentation-du-public-dans-la-salle-quelques-considérations, consulté le 4 mai 2020.

18. Michel Serres, The Parasite [1980], trad. Lawrence R. Schehr, Minneapolis, University of Minnesota Press, 2007, p. 224-234 ; Bruno Latour, We Have Never Been Modern, trad. Catherine Porter, Cambridge (MA), MIT Press, 1993, p. 225. 19. Bruno Latour, Reassembling the Social. An Introduction to the ActorNetwork-Theory, Oxford, Oxford UP, 2005. NdT : L'ouvrage est paru en français aux éditions La Découverte en 2006 sous le titre Changer de société. Refaire de la sociologie. $\doteq$

20. Bruno Latour, Changer de société, op. cit., p. $8 . \pm$

21. Ibid., p. 67. $\bullet$

22. Margaret Werry, «Interdisciplinary Objects, Oceanic Insights. Performance and the New Materialism », dans Henry Bial et Scott Magelssen (dir.), Theatre Historiography. Critical Interventions, Ann Arbor, University of Michigan Press, 2010, p. 222 et 226.

23. Marvin Carlson, The Haunted Stage. The Theatre as Memory Machine, Ann Arbor, University of Michigan Press, 2003 ; Rebecca Schneider, Performing Remains. Art and War in Times of Theatrical Reenactment, Londres, Routledge, 2011. $ヒ$

24. Bruno Latour, op. cit., p. 166.

25. Idem. $\bullet$

26. John Wall, «Transforming the Object of our Study. The Early Modern Sermon and the Virtual Paul's Cross Project », Journal of Digital Humanities [en ligne], $\mathrm{n}^{\circ} 3-1$, printemps 2014, http://journalofdigitalhumanities.org/3-1/transforming= the-object-of-our-study-by-john-n-wall, consulté le 4 mai 2020 . 
27. John Moyer, «John Donne delivered a Sermon on Gunpowder Day in 1622. What did it sound like ? », Humanities. The Magazine of the National Endowment for the Humanities [en ligne], $\mathrm{n}^{\circ} 35-5$, sept.-oct. 2014, https://www.neh.gov/humanities/2014/septemberoctober/curio/digital-humanitiesproject-recreates-what-john-donne-sounded-, consulté le 4 mai 2020. 28. Matt Azevedo, Ben Markham et John N. Wall, « Acoustical Archaeology. Recreating the Soundscape of John Donne's 1622 Gunpowder Plot sermon at Paul's Cross », Acoustical Society of America. Proceedings of Meetings on Acoustics [en ligne], $\mathrm{n}^{\circ} 133-5,2013$, https://asa.scitation.org/doi/10.1121/1.4799054, consulté le 4 mai 2020. 29. « Overview », Virtual Paul's Cross Project, https://vpcpp.chass.ncsu.edu, consulté le 4 mai 2020. $匚$ 30. «About Rome reborn», http://romereborn.frischerconsulting.com:80/about.php, consulté en juillet 2017. Ce site disparu peut désormais être consulté sur https://archive.org. $\_$ 31. «About Us », https://www.romereborn.org/content/aboutcontact, consulté le 4 mai 2020.

32. Jonathan Westin et Ragnar Hedlund, « Polychronia. Negotiating the Popular Representation of a Common Past in Assassin's Creed », Journal of Gaming \& Virtual Worlds, $\mathrm{n}^{\circ} 8-1$, mars 2016, p. 17. 33. Ibid., p. $4 . \pm$

34. Adam Chapman, «Is Sid Meier's Civilization History ? », Rethinking History, $\mathrm{n}^{\circ} 17-3,2013$, p. 315.

35. https://sites.google.com/site/louisbretez/home, consulté le 4 mai 2020. 36. Laure Cailloce, «Écoutez le Paris du XVIII ${ }^{\mathrm{e}}$ siècle », CNRS. Le journal [en ligne], juillet 2015, https://lejournal.cnrs.fr/articles/ecoutez-le-paris-du-xviiiesiecle, consulté le 4 mai 2020.

37. Mylène Pardoen, «Archi'sons : la valorisation d'archives par le sonore », dans Valoriser les archives, diffuser les savoirs : dynamiques de partage et méthodes innovantes, Marseille, Association des étudiants et diplômés en archivistique d’Aix-Marseille Université, 2014, 
https://www.dropbox.com/s/hjwhjet9nol30dc/aedamu_je3 publi actes_pardoen vf.pdf, consulté le 4 mai 2020. 38. Michael Hawcroft, « Comment jouait-on le rôle d’Hippolyte dans la Phèdre de Racine ? », XVII siècle, $\mathrm{n}^{\circ}$ 231-2, 2006, p. 243. Pour une histoire de ce projet et de ses enjeux, voir Céline Candiard, « Le théâtre baroque aujourd'hui en France : la musique pour modèle », Littératures classiques, n 91-3, 2016, p. 153161.

39. Ces modèles ont une visée différente de celle des histoires spéculatives des Lumières, parmi lesquelles on trouve le Discours sur l'origine et les fondements de l'inégalité de Rousseau qui imagine, sans pouvoir s’appuyer sur un quelconque document, les premiers moments de la société - tout comme Le Début de l'histoire, des origines de la politique à nos jours (2011) de Francis Fukuyama nous pousse à imaginer un monde que nous ne pouvons pas connaître, en continuité avec le nôtre. $ヒ$

40. Voir, respectivement, Joël Schmidt, La Saint-Barthélemy n’aura pas lieu, Paris, Albin Michel, 2011 ; Guillaume de Bertier de Sauvigny, «A Trip to The Galaxy of "if" », Consortium on Revolutionary Europe 1750-1850. Proceedings, vol. 6, 1977, p. 84-91 ; David S. Landes, « What Room for Accident in History ? Explaining Big Changes By Small Events », Economic History Review, n 47-4, 1994, p. 637-656.

41. John N. Wall, « Recovering Lost Acoustic Spaces : St Paul's Cathedral and Paul's Churchyard in 1622 », Digital Studies/le Champ Numérique [en ligne], n 3-3, 2014, https://www.digitalstudies.org//article/10.16995/dscn.58, consulté le 4 mai $2020 . \triangleq$

42. Laurence Marie, «L'entrée de Shakespeare au répertoire de la ComédieFrançaise au XVIII ${ }^{\mathrm{e}}$ siècle », Littératures classiques, n 95, 2018, p. 181-190. 43. Jeffrey S. Ravel, « La Comédie-Française par les chiffres, 1752-2020 » et Jeffrey Peters, « Voir le littéraire : la visualisation de données et le Projet des

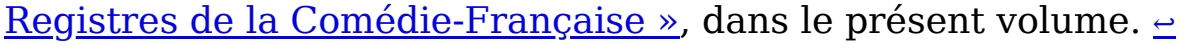
44. DARTH (Digital Arts and Humanities), «Visualisation des billets vendus », septembre 2016, http://darthcrimson.org/visualisation-des-billets-vendus, consulté le 4 mai $2020 . \triangleq$ 
45. Voir Pierre-Alain Clerc, " Le "débit" de la déclamation aux XVI ${ }^{\mathrm{e}}$ et XVIII siècles », dans Xavier Bisaro et Bénédicte Louvat-Molozay (dir.), Les Sons du théâtre..., op. cit., p. 215-237 ; et Sabine Chaouche, L'Art du comédien. Déclamation et jeu scénique en France à l'âge classique, Paris, Honoré Champion, 2001. Pour un aperçu de cette recherche, voir Julia Gros de Gasquet, «Rhétorique, théâtralité et corps actorial », $X V I I^{e}$ siècle, $\mathrm{n}^{\circ} 236,2007-3, \mathrm{p} .501$ 519.

46. "Outcomes », https://vpcp.chass.ncsu.edu/outcomes/, consulté le 4 mai 2020. Voir Bruce Reed, The Dynamics of Religion. Process and Movement in Christian Churches, Londres, Darton, Longeman et Todd, 1978.

47. Georges Forestier, Notice d'Athalie dans Jean Racine, Fuvres complètes, éd. Georges Forestier, Paris, Gallimard, 1999, p. 173. 48. Renaud Bret-Vitoz, L'Espace et la Scène. Dramaturgie de la tragédie française, 1691-1 759, Londres, Voltaire Foundation, 2008, p. 28. 49. Derek Miller, «Four Perspectives on the Comédie-Française Repertoire », juin 2016, https://www.cfregisters.org/cf-repertoire, consulté le 4 mai 2020. 50. En d'autres termes, Athalie ne figure pas au répertoire de 21 années : elle n'est pas jouée de 1718 à 1720, en 1724, en 1726-27 et en 1731. À partir de 1733, elle n'apparaît plus sur la scène pendant cinq ans. Durant les années 1740, elle est absente en 1749 seulement, puis, dans les décennies suivantes, en 1752, en $1760,1763,1765,1772$ et $1776 . \subseteq$

51. Voir Junga Shin, «La construction d'un "Racine classique" au XVIII ${ }^{\mathrm{e}}$ siècle », dans Nicholas Cronk et Alain Viala (dir.), La Réception de Racine à l'âge classique. De la scène au monument, Oxford, Voltaire Foundation, 2005. Elle retrace la représentation que l'on se faisait de Racine au XVIII ${ }^{\mathrm{e}}$ siècle. $ヒ$ 52. Sur ces actrices et le type de rôles qui leur étaient attribués, voir Virginia Scott, Women on the Stage in Early Modern France, 1540-1750, Cambridge, Cambridge UP, 2018, p. 205-206. 53. Georges Forestier, Notice d'Athalie, op. cit., p. 178.

54. Renaud Bret-Vitoz, op. cit., p. 245.

55. Bruno Latour, op. cit., p. 166. 
56. Michel Chion, La Voix au cinéma, Paris, Éditions de l’Étoile/Cahiers du Cinéma, 1982.

57. L'auteur remercie sincèrement Christophe Wall-Romana ainsi que Tom Conley, David Franco, Jeffrey Peters et Ellen Welch pour leurs commentaires au fil du développement de ce projet. Elle est particulièrement reconnaissante à l'égard de Sylvaine Guyot et de Jeffrey Ravel pour leurs encouragements. $\leftrightarrows$ 